\title{
Motivaciones hacia la Responsabilidad Social en las PYMEs familiares
}

\section{Drivers towards CSR practices in family firms}

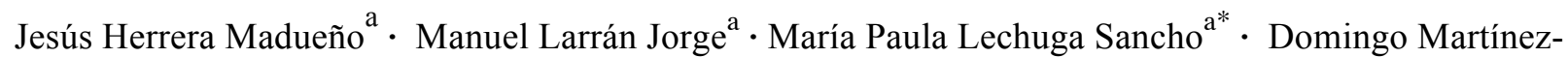 \\ Martínez $^{\mathrm{a}}$ \\ ${ }^{a}$ Facultad de Ciencias Económicas y Empresariales de la Universidad de Cádiz (Cádiz). Los autores aparecen en \\ estricto orden alfabético.
}

D A TOS ARTÍC U L O

Historial:

Recibido 11-12-2013

Aceptado 05-04-2014

Palabras clave:

Responsabilidad Social de la Empresa

Empresas familiares

Motivaciones

Barrerass

Códigos JEL:

M14

\section{A R T I C L E IN F O}

Article history:

Received 11-12-2013

Accepted 05-04-2014

Keywords:

Corporate Social

Responsibility

Family Firms

Drivers

Barriers

JEL codes:

M14

\section{R E S U M E N}

Las prácticas Responsabilidad Social Empresarial (RSE) se han configurado como un área de interés entre los académicos e investigadores. Sin embargo, la mayor parte de estudios se centran en organizaciones de mayor dimensión, siendo escasos los trabajos centrados en las pequeñas y medianas empresas (Pymes) y más concretamente en el ámbito de las empresas familiares (EF). Con estas premisas, el presente trabajo trata de analizar si existen comportamientos diferenciales entre las Pymes familiares versus no familiares y como éstos pueden estar influidos por factores tales como el tamaño, el género y el nivel de estudios reglados. Los resultados señalan que no existen grandes diferencias, si bien, la visión a largo plazo de las EF hace que estas integren, en mayor medida, los aspectos sociales y ambientales en la estrategia.

\begin{abstract}
A B S T R A C T
The field of corporate social responsibility has grown exponentially in the last decade. These practices have been configured as an area of interest among academics and researchers. However, most studies focus on larger organizations, with few studies focusing on small and medium enterprises (SMEs) and more specifically in the field of family businesses. With these premises, this paper seeks to analyze whether there are differential social responsibility behavior between family business and not familiar business, and if they may be influenced by factors such as size, gender and level of formal studies. The results indicate that there are no major differences, though, and given the long-term vision of family business, they do incorporate social and environmental aspects in their strategy.
\end{abstract}




\section{Introducción}

Actualmente, la sociedad no percibe a la empresa únicamente desde una perspectiva financiera, ya que los cambios provocados por el entorno, la mayor globalización de los mercados, la mayor repercusión social que ésta tiene, la contribución al desarrollo de las comunidades en las que opera mediante la creación de riqueza, así como otros factores tales como los medioambientales, la necesidad de incorporar prácticas de buen gobierno o la de llevar a cabo inversiones socialmente responsables, entre otros, han motivado a las organizaciones a adoptar cambios en su gestión mediante la implementación de prácticas socialmente responsables (Moneva y Hernández, 2009).

Sin embargo, aún cuando el sentido social de las organizaciones se está convirtiendo en un factor clave para el funcionamiento, mantenimiento $\mathrm{y}$ supervivencia a largo plazo de las mismas (Muñoz y Cabaleiro, 2009), la adopción de dichas prácticas no ha tenido un desarrollo similar entre las grandes corporaciones y las pequeñas y medianas empresas (en adelante, Pymes). Ello se ha debido fundamentalmente a las diferencias propias que presentan éstas últimas en cuanto a su estructura de la propiedad, a las características del empresario y al ámbito local en el que operan, entre otras cuestiones (Jenkins, 2004; Herrera et al., 2013). En cualquier caso, estos rasgos característicos afectan a los contenidos, a la naturaleza y al alcance de las actividades de responsabilidad social empresarial (en adelante, RSE), desarrollándose así una nueva interpretación de la misma, adaptada a las características y peculiaridades de este tipo de organizaciones (Guibert, 2009).

En este contexto, no debemos olvidar que la gran mayoría de las Pymes son de carácter familiar y no sólo en nuestro país sino también en el ámbito internacional. La importancia de la empresa familiar dentro de la economía y el mundo de la empresa en general es incuestionable (Cabrera et al., 2005; Casado y Rodríguez, 2009; Long y Mathews, 2011). Más concretamente, en España, existen 2,9 millones de empresas familiares (en adelante, EF), que representan el $70 \%$ del PIB español y generan aproximadamente $60 \%$ de los empleos (GarcíaBorbolla et al., 2009).

Aunque dentro del ámbito familiar Cambell (2007) incide en la ausencia de trabajos que analicen las razones que mueven a las empresas a actuar o no de una forma socialmente responsable, sí que existen ya ciertas evidencias. Así por ejemplo, Uhlaner et al., (2004) sugieren que las EF son más propensas a tener una relación especial con los empleados debido al carácter familiar de la empresa. Por otro lado, estudios previos ponen de manifiesto que las características distintivas de las EF son un acelerador hacia la consecución de prácticas socialmente responsables (Uhlaner et al., 2004; Deniz y Suarez, 2005; Block, 2010), concretamente Graafland (2002) demostró que el nivel de preocupación de las EF por la RSE es mayor que el de las no familiares. Esto se puede deber a sus características particulares; estas empresas tienen una serie de condiciones intrínsecas que promueven una predisposición natural a integrar la RSE en su gestión diaria (Casado y Rodríguez, 2009).

A pesar de la relevancia económica y de las particularidades de las $\mathrm{EF}$, se ha prestado poca atención a investigar su naturaleza y funcionamiento (Herrera et al., 2012) y es por ello que autores como Dyer y Whetten (2006) expongan la necesidad de una investigación más profunda para "explorar y entender las condiciones o factores que motivan a las EF a apoyar las iniciativas de RSE" (Dyer y Whetten, 2006). En este contexto, tradicionalmente se ha identificado a las EF con determinadas características que podrían incidir tanto positiva como negativamente sobre las relaciones que este tipo de entidades mantienen con sus stakeholders, asociaciones que, por otro lado, podrían además estar ligadas a diferentes orientaciones hacia la RSE (Cabrera et al., 2005; Bingham et al., 2011).

A partir de lo anterior, el primer objetivo del presente trabajo es el análisis de la existencia o no de diferencias significativas en las motivaciones y barreras que perciben tanto las pequeñas y medianas empresas familiares como no familiares. Una vez analizado esto, el segundo objetivo de este trabajo se centra en el estudio de la incidencia que determinados factores explicativos como el tamaño, el género y la formación universitaria del propietario gerente tienen sobre las motivaciones y barreras percibidas por las Pymes familiares.

Para su consecución, se han identificado a partir de la literatura previa los principales aceleradores y barreras hacia la RSE de estas empresas de menor dimensión. Una vez seleccionadas se exponen las hipótesis a contrastar en relación al carácter familiar y algunas de las características organizativas de las Pymes típicamente asociadas al comportamiento sostenible. Posteriormente, se expone el procedimiento metodológico seguido para la ejecución del estudio empírico y para el contraste de las hipótesis propuestas. Por último, se identifican los principales resultados y se prosigue con una 
discusión sobre los mismos, identificando las principales conclusiones y señalando algunas posibles limitaciones al estudio.

\section{Motivaciones y barreras implementación de la RSE en Pymes}

para la

Como tradicionalmente se ha puesto de manifiesto, las Pymes se han caracterizado por contribuir de forma activa al desarrollo de las comunidades en las que opera, no sólo desde una perspectiva cuantitativa, en cuanto a que constituye la estructura más frecuente del tejido industrial de cualquier economía, sino también cualitativa, a través de la generación de empleo y riqueza o impulsando la investigación y el desarrollo entre otros (Fox, 2005). Este tipo de organización no es ajena al contexto en el cual se inserta siendo en gran medida responsables de la sostenibilidad de su entorno. Debido a ello, y dado que actualmente no es suficiente con tener una economía sostenible sino que se ha de asegurar su propio equilibrio sostenible a largo plazo, las Pymes han de encontrar un balance entre el desarrollo económico, el progreso social y la protección del medioambiente pese al problema general de escepticismo acerca de las implicaciones prácticas del concepto de RSE que se ve reforzado por la aguda crisis que ocupa toda la atención de los ciudadanos, las empresas y los políticos.

En tiempos de crisis, la inclusión de la responsabilidad y el respeto a la ley debe ser más que nunca una realidad en todos los tipos de organizaciones: pequeñas, medianas y grandes (Williamson et al., 2006; Moore y Spence, 2006). La ética y la transparencia son temas cada vez más demandados a nivel global como resultado de una necesidad de confianza, justicia social y respeto a futuras generaciones.

Partiendo de las diferencias propias de las pequeñas empresa en relación a las grandes, de las acepciones que han manifestado numerosos autores en relación a la necesidad de elaborar un modelo de RSE propio para las empresas de reducida dimensión (Jenkins, 2004; Sweeny, 2007), y teniendo en cuenta que se trata de un concepto aún no consolidado, para mejorar el aprendizaje y la capacidad de implementar prácticas de RSE en las Pymes hemos considerado necesario la determinación de los factores (limitadores o motivadores) que influyen en mayor o menor medida en estas empresas más pequeñas y que se han de tener en cuenta para comprometerlas con la RSE. En este sentido, parece conveniente determinar qué factores y/o razones, positivas o negativas influyen en mayor $o$ menor medida en el compromiso que este tipo de organizaciones adopta de cara a la RSE.

Según se puede observar, los estudios empíricos previos muestran cómo los motivos que explican la inactividad de muchas Pymes en el campo de la RSE se refieren principalmente a: la falta de información y conocimiento sobre ésta y el modo en que la integran en sus actividades, la falta de recursos humanos y materiales, y las dificultades para convertir los esfuerzos sociales y medioambientales en beneficios tangibles. Sin embargo, no hemos de olvidar que la implantación de políticas en esta materia puede conllevar significativas ventajas competitivas que acaben incrementando el valor de la empresa en la medida que un desempeño sostenible puede acarrear mejoras asociadas con la reputación, la gestión de riesgos, el reclutamiento de empleados, la motivación y retención de los mismos, mejoras en sus relaciones con inversores y con el acceso al capital, mayores niveles de aprendizaje e innovación, mejoras en la competitividad y en el posicionamiento en el mercado, incremento de la eficiencia operativa, etc.

En el Anexo 1 se resumen y presentan los principales inconvenientes que limitan el desarrollo de prácticas de RSE por parte de estas empresas.

Las cuestiones sociales o medioambientales no deben presentarse o ser percibidas como una carga o barrera para las Pymes, sino como un deber social y deseable a nivel individual. Tanto a través de numerosas iniciativas públicas o privadas como en la literatura de Pymes existen argumentos a favor del aumento de la implicación e implantación de medidas de RSE: mejor alineamiento con las expectativas de la ciudadanía; mejora del clima laboral y de la productividad y motivación de los trabajadores; aumento de las relaciones con los stakeholders; aprendizaje e innovación; posibilidad de desarrollar alianzas estratégicas con otros agentes (Borga et al., 2009; Gelbmann, 2010). Es por ello que se considera interesante la determinación de los diferentes aceleradores o factores sobre los que instituciones gubernamentales y otros interesados podrían incidir a fin de conseguir una mayor atención y aceptación a esta cuestión por parte de las Pymes.

En el Anexo 2 se muestra los principales aceleradores que inciden en la puesta en práctica de actividades de RSE en las Pymes. 
Si bien no se han encontrado en la literatura trabajos específicos en los que se determine las principales barreras y aceleradores que llevan a las EF a la consecución de prácticas socialmente responsables, estudios previos sugieren que la propiedad familiar, por sus características distintivas, puede influir de forma positiva en la adopción de las políticas de RSE (Stavrou et al., 2007; Block, 2010; Amann et al., 2011). El principal argumento en apoyo de esta afirmación se sustenta en la noción de reputación corporativa (Block, 2010; Wagner, 2010; Amann et al., 2011). Por lo general, en comparación con las empresas no familiares (en adelante, ENF), los propietarios de las EF sienten un mayor grado de identificación con la organización y buscan preservar la empresa como herencia familiar para su transmisión a las siguientes generaciones (Abdellatif et al., 2010). Esto, conduce a los gestores familiares a mostrar una mayor preocupación por la reputación de sus respectivas empresas, evitando cualquier tipo de acción nociva para la imagen corporativa (Uhlaner et al., 2004; Wagner, 2010), y estableciendo políticas más sólidas de inversión a largo plazo.

Más allá de este argumento sobre la reputación corporativa, la literatura proporciona explicaciones complementarias adicionales, como pudiera ser la forma en que la propiedad familiar facilita el control efectivo de la gestión (Ward, 2004). A menudo, los propietarios de la familia tienen un profundo conocimiento de la empresa y, por ende, de sus actividades de negocio, lo que contribuye a reducir las posibles asimetrías informativas y facilita un control eficaz. En este sentido, los propietarios gerentes de una empresa familiar deberían estar más motivados por llevar a cabo una eficaz supervisión, ya que se les presupone emocionalmente conectados con su empresa y con las actividades desarrolladas en la misma (Zellweger y Astrachan, 2008).

Otra diferencia importante entre las empresas familiares y no familiares es que la estructura de propiedad de la empresa familiar asegura una alineación efectiva entre los objetivos de la empresa y sus propietarios (Schulze et al., 2001; Astrachan et al., 2003; Uhlaner, 2004), estando esto relacionado tanto con aspectos éticos (Brice, 2013), como de desempeño social (Déniz y Suárez, 2005) y ambiental (Walls et al., 2007). En este sentido, la estructura de la empresa familiar reduce los problemas de agencia entre propietarios y directivos, marco teórico que ha sido utilizado en la literatura empresarial para justificar el compromiso social de este tipo de organizaciones. En particular, se considera que uno de los principales factores que explican la incorporación de prácticas de RSE es el relacionado con la estructura de la propiedad de las empresas, en la medida que, cuanto menor sea la separación entre la propiedad y el control, como es el caso de las EF, mayor será el incentivo a la consecución de prácticas que atiendan los intereses de los diversos stakeholders (Webb, 2004).

Igualmente, Uhlaner (2004) defiende que las EF son más propensas a tener más fortalezas y menos debilidades hacia la RSE que otras empresas, ya que normalmente sus objetivos van más allá de la maximización del beneficio económico. Igualmente, por regla general, a las EF se les atribuye una relación más cercana con sus empleados y con sus clientes que la que llegan a mantener las ENF.

En este contexto de interacciones, la perspectiva teórica más empleada y que permite explicar la predisposición sostenible de las empresas de carácter familiar es la teoría de los stakeholders (Uhlaner et al., 2004; Bingham et al., 2011). Zellweger y Nason (2008) explican cómo las EF tienen un mayor sentido de obligación hacia las demandas de un comportamiento socialmente responsable por parte de los diferentes stakeholders. En comparación con las empresas no familiares, por ejemplo, las familiares tienen un incentivo particular para tener una perspectiva más a largo plazo y conseguir perpetuar su negocio para las futuras generaciones o relevo generacional (Bingham et al., 2011). De esta manera, las EF pueden desarrollar lazos emocionales más fuertes con las partes interesadas (Sirmon y Hitt, 2003). En este mismo sentido, las EF son más propensas que las no familiares a ver a sus stakeholders como socios. Debido a que este tipo de colaboración requiere confianza, responsabilidad y el compromiso de tiempo y recursos, las EF tienden a alinearse con aquellos stakeholders, cuyos valores e ideales se identifican con los atributos centrales, distintivos y perdurables de la empresa (Bingham et al, 2011). Para la mayoría de las EF, esta perspectiva relacional incluiría una mayor participación en la comunidad local, un mayor respeto, una mejora del bienestar de sus empleados y un enfoque estratégico a largo plazo fundamentado en ideales relacionados con la tradición y reputación de sus productos o servicios (Miller y Le BretonMiller, 2003; Van Buren, 2005).

Por todo ello es lógico pensar que las empresas no familiares son menos propensas que las familiares a la implementación de prácticas de RSE y que, por tanto, el carácter familiar podría ser determinante en la adopción que las Pymes hagan de 
la RSE, incidiendo sobre las motivaciones y barreras hacia la misma.

H1: Las Pymes familiares perciben mayores motivaciones y menores barreras hacia la RSE que las no familiares.

Si bien el carácter familiar ha sido señalado habitualmente como una de las características organizativas que hace que las empresas sean más proclives al fomento de la RSE, no todas las EF se comprometen de igual modo (Uhlaner, 2004). Así, por ejemplo, algunas de las características más analizadas, como el tamaño, el género y formación de su propietario o gerente podrían ser la clave de los distintos perfiles de orientación hacia la RSE.

En el marco de las grandes corporaciones son abundantes los trabajos que han analizado la influencia que pueden ejercer determinadas características asociadas a la propia empresa o a la figura de su máximo responsable sobre el desarrollo de las prácticas de responsabilidad social (De Jong et al., 2008; Nielsen y Thomsen, 2009; Godos-Díez et al., 2011). Concretamente, es la figura del director de las grandes empresas la que mayor atención ha recibido en cuanto al desarrollo de modelos que expliquen hasta qué punto determinadas características de los mismos pueden influir en sus creencias éticas y por tanto, en una toma de decisiones más o menos éticas (Herrera et al., 2012). Así, el compromiso sostenible de las empresas depende directamente tanto de la alta administración como del entorno interno o externo de las mismas (López-Gamero et al., 2008). En base a lo anterior, estos trabajos, por lo general, se basan fundamentalmente en la teoría de la elección estratégica (Child, 1972) y en la teoría de los escalones superiores (Hambrick y Manson, 1984) para determinar que las decisiones de gestión pueden interpretarse como el reflejo de los atributos y características personales de los miembros de la alta dirección.

En este sentido, es preciso señalar que en dichos estudios se distingue fundamentalmente entre factores inherentes e individuales adquiridos. Entre los factores inherentes destacan aquellos asociados a los atributos personales como el género (SimgaMugan et al., 2005; Strautmanis, 2008). La participación ya frecuente de las mujeres en las empresas ha suscitado interés entre los investigadores por examinar las variaciones de género en lo que respecta a las consideraciones éticas y de responsabilidad social de las empresas dado que existe evidencia que sugiere que las mujeres suelen ser más éticas que los hombres (Hazlina y Seet, 2010). Aunque conscientes de que la presencia femenina en puestos de dirección es bastante escasa en el mercado de trabajo español, entendemos que las características del personal directivo son diferentes dependiendo de su género $y$, por ende, que existen diferencias de género en el estilo de dirección que inciden en la forma en que cada directivo gestiona la consecución de los objetivos, las estrategias de cambio y las actividades en las que se involucra. Así por ejemplo, a las mujeres directivas se les atribuye un liderazgo más interactivo, orientado a estimular la participación y la autoestima de los demás (Rosener 1990 y 1995). Además, a las mujeres se les presupone tener un instinto más filantrópico y una mayor sensibilidad social (Burgess y Tharenou, 2002) que a los hombres, a quienes por el contrario se les atribuye un instinto mucho más económico (Burgess y Tharenou, 2002) o asocia con mayor frecuencia una racionalidad económica superior, $\mathrm{y}$ por tanto conductas mucho más reactivas, concentradas en los objetivos y el control (Vinnicombe, 1995). En definitiva, las mujeres parecen tener una visión más holística de la realidad, siendo esta un aspecto clave requerido por la RSE (De Miguel, 2011). Otra evidencia empírica relacionada con lo anterior, se pone de manifiesto en el estudio centrado en Pymes de Hazlina y Seet (2010) quienes muestran como las mujeres perciben la ética y la responsabilidad social como más importantes que sus homólogos masculinos en la gestión de su negocio.

Este factor explicativo hacia las prácticas de RSE parece obtener mayor relevancia cuando se habla de $\mathrm{EF}$, donde el hecho de que una empresa favorezca una orientación más o menos amplia hacia la RSE dependerá en mayor medida de las características intrínsecas del propietario o gerente, de la familia y de su visión sobre la naturaleza humana (Salvato, 2002). A partir de estos argumentos, se plantea la siguiente hipótesis de trabajo:

H2: Las Pymes familiares que cuentan con un propietario gerente mujer perciben mayores motivaciones y menores barreras que las que tienen un propietario gerente hombre.

Por otro lado, uno de los factores adquiridos más estudiados ha sido el que tiene que ver con la educación (Granitz, 2003; Fritzsche y Oz, 2007). Sin duda, la formación universitaria o superior incide sobre la deontología del individuo, orientando la definición de la ética profesional de cada disciplina e 
incluso su rol social. A partir del trabajo de Besser (1998), Fitzgerald et al. (2010) contrastaron que los individuos con mayor nivel de estudios reglados eran más proclives a participar como líderes de organizaciones de carácter social y local. Igualmente, Dawson et al., (2002) estudiaron la influencia que la educación tiene sobre la actitud en el caso de propietarios de microempresas de Australia, comprobándose que estos otorgaban una mayor importancia al comportamiento ético en su actividad diaria a medida que aumentaba el nivel de estudios.

De todo lo anterior y basándonos en otros estudios encontrados que analizan la actitud ética en los negocios como variable dependiente de determinados rasgos personales, tales como el género (Serwinek, 1992; Hazlina y Seet, 2010) o el nivel de estudios del propietario gerente (Fitzgerald et al., 2010), proyectamos la siguiente hipótesis:

H3: Las Pymes familiares que cuentan con un propietario gerente con cualificación universitaria perciben mayores motivaciones y menores barreras que las que tienen un propietario gerente sin estudios universitarios.

Así, además de los factores inherentes e individuales adquiridos por los propietarios gerentes, pueden considerarse como determinantes de la adopción de prácticas de RSE otras características intrínsecas a la propia naturaleza de cada empresa. En este sentido uno de los factores explicativos analizados con mayor frecuencia por su posible incidencia sobre el comportamiento sostenible de las organizaciones ha sido el tamaño.

Ya en 1973, Eilbirt y Paket encontraron que el tamaño era un factor que estaba positivamente asociado con la participación de las empresas en la responsabilidad social. A partir de entonces, en la literatura de RSE en general, se considera que existe una relación directa entre el tamaño de la empresa y una mayor implicación y comprensión del concepto integral de la RSE (Udayasankar, 2008; Bajo y Durán, 2009; Güler et al., 2010). En este sentido, más concretamente en algunos estudios relativos a la RSE en Pymes el tamaño es analizado como un factor explicativo de las decisiones específicas que atañen a la misma $\mathrm{y}$, por tanto, condiciona la cantidad de actividades sostenibles (Vives et al., 2005; Lepoutre y Heene, 2006; Perrini et al., 2007; Russo y Tencati, 2009; Preuss y Perschke, 2010), siendo las de tamaño micro y pequeñas las que menos recursos destinan a estas prácticas quizás debido a la falta de codificación de la responsabilidad social en las pequeñas empresas (Spence, 1999; Jenkins, 2004).

En este contexto, la teoría de la legitimidad conforma el marco necesario para justificar los argumentos que relacionan un mayor tamaño empresarial con una mayor predisposición a adoptar prácticas de RSE. Las organizaciones más grandes son observadas más de cerca y están sometidas en mayor medida al escrutinio público y a fuertes presiones sociales (Fassin, 2008). Williamson y Lynch-Wood (2008) ponen de manifiesto cómo toda empresa, para asegurarse la existencia en el largo plazo, debe intentar atender las expectativas de sostenibilidad y buen hacer de su comunidad y de otros grupos de interés. En este sentido, cabría esperar que las entidades de gran dimensión, con el fin de proyectar una imagen de empresa responsable y legitimar así su actuación frente a la sociedad, desarrollen más prácticas de RSE. Sin embargo, a diferencia de las grandes empresas, las Pymes, al operar en mercados locales y depender en gran medida de fuentes internas para financiar su crecimiento, tienen una visibilidad mucho más limitada y no sienten la misma presión por la formalización de sus prácticas. En general, estas empresas de reducida dimensión se caracterizan por tener una menor documentación de sus operaciones, menos obstáculos procedimentales y por aplicar un estilo de gestión informal a las cuestiones estratégicas, incluyendo la RSE (Russo y Tencati, 2009). Estos autores afirman que existe una asociación directa entre el tamaño organizativo y el nivel de formalización de las estrategias sostenibles de las empresas. La RSE puede verse, por tanto, como la impresión simbólica o imagen construida de una empresa, por medio de la cual, ésta se comunica con el exterior para dar a conocer su cultura corporativa y controlar tanto su posición económica como política.

A tenor de las evidencias proporcionadas, la última hipótesis a contrastar en este trabajo será la siguiente:

H4: Las empresas familiares medianas perciben mayores motivaciones y menores barreras que las pequeñas.

\section{Metodología}

\subsection{Selección de la muestra}

La selección de las empresas se realizó de forma aleatoria en base al listado SABI de empresas de la 
Tabla 1

Análisis de los datos muestrales.

Datos Poblacionales

Datos Muestrales

Muestra final

\begin{tabular}{lllllll}
\hline Tamaño: & Recuento & $\%$ & Recuento & $\%$ & Recuento & $\%$ \\
\hline Pequeñas & 5.810 & 87,77 & 3.013 & 87,56 & 355 & 73,80 \\
Medianas & 809 & 12,23 & 428 & 12,44 & 126 & 26,20 \\
\hline Total & 6.619 & 100 & 3.441 & 100 & 481 & 100 \\
\hline Fuente: Elaboración propia a partir de los datos del DIRCE 2009
\end{tabular}

Fuente: Elaboración propia a partir de los datos del DIRCE 2009

región de Murcia. De las entidades listadas, se realizó una selección en base a una frecuencia elegida al azar y a partir de una tabla de números aleatorios. No obstante, se establecieron los siguientes criterios de selección:

- Selección de empresas con 20 o más trabajadores.

- Selección de empresas en función del sector y rama de actividad. Para ello, con el fin de asegurar la representatividad de la muestra, se fijaron unas cuotas mínimas tomando como referencia el número de empresas pertenecientes a las distintas ramas de actividad empresarial de la región.

El proceso de recogida de información fue realizado directamente por la empresa Consultores CSA, quienes se encargaron de contactar con cada una de las Pymes seleccionadas. Si bien la muestra inicial ascendía a 552 empresas, tras el proceso de filtrado, por el que se eliminaron aquellos cuestionarios erróneamente cumplimentados, los cuestionarios de empresas con menos de 10 empleados (microempresas) y los cuestionarios de aquellas organizaciones que tuviesen una forma jurídica que, como las sociedades cooperativas, requiriesen un tratamiento específico, se acabó reduciendo la muestra a 481 empresas (Tabla 1).

\subsection{Diseño y validez del cuestionario}

La información sobre las variables necesarias para el estudio fue recabada a través de un cuestionario dirigido a los gerentes de la empresa, o bien a los mandos intermedios responsables del área de responsabilidad social. Siguiendo las recomendaciones de De Vaus (1993), se realizó una fase de testeo real del cuestionario y de la metodología escogida a través de la aplicación de 15 entrevistas previas. De las observaciones del mismo, se implementaron mejoras como la adecuación del lenguaje utilizado o la elaboración de instrucciones más específicas para los encuestadores, los cuales recibieron formación sobre la RSE y la aplicación del cuestionario. El trabajo fue llevado a cabo entre los meses de diciembre de 2010 y febrero de 2011 .

Las cuestiones consideradas en el cuestionario final fueron medidas principalmente a través de una escala Likert de cinco puntos conforme a lo establecido en el trabajo de Maxhaam III y Netemeyer (2003). Este tipo de escala ha sido ampliamente utilizada en la metodología de encuesta llevada a cabo por investigadores en trabajos previos sobre RSE y Pymes (Zhu et al., 2008; Baden et al., 2009).

\subsection{Estadísticos empleados para el contraste de hipótesis}

Para el contraste de hipótesis se han utilizado dos procedimientos estadísticos distintos en base a la naturaleza de las variables a contrastar, el Chicuadrado de Pearson y la U de Mann-Whitney.

En aquellos casos en los que la relación a contrastar ha sido entre variables categóricas, además de observar las frecuencias en una tabla de contingencias, se ha empleado como estadístico para medir la posible asociación el Chi-cuadrado de Pearson. Mediante la comparación entre las frecuencias esperadas y las observadas esta prueba permite contrastar la hipótesis nula de que las variables son independientes.

Por otro lado, para aquellos contrastes de hipótesis en los que se consideraban variables cuantitativas y la categórica, empresa familiar o no, se comprobó en primer lugar si los datos seguían o no una distribución normal. Para ello y tratándose de una muestra de más de 50 casos se optó por la prueba de Kolmogorov-Smirnov. Tras confirmar que los datos no seguían una distribución normal ni aún haciendo una transformación logarítmica de los mismos, se optó por la prueba no paramétrica $U$ de Mann-Whitney, por ser considerada como la que mejor se ajusta a los objetivos de nuestra investigación. Dicha prueba basa su hipótesis nula en considerar que las distribuciones de probabilidad 
de las que se extraen las muestras son idénticas aun siendo desconocidas, y la hipótesis alternativa de que las distribuciones son diferentes. Dicho de otro modo, la hipótesis nula se basa en demostrar si las muestras tomadas independientemente provienen de poblaciones que tienen la misma media, mientras que la alternativa puede establecer que las esperanzas matemáticas son diferentes (contraste de dos colas), o que una de ellas, previamente escogida, es superior a la otra (contraste de una cola).

\subsection{El carácter familiar de las empresas de la muestra}

En la literatura previa existen diversas definiciones de empresa familiar, no existiendo un consenso general en torno a cómo determinar el carácter familiar (Herrera et al., 2011). No obstante, Villalonga y Amit (2004) y Allouche et al. (2008) sugieren que la mayoría de las definiciones incluyen por lo menos tres dimensiones: (i) una o varias familias deben tener una parte importante de la capital, (ii) los miembros de la familia deben conservar control significativo sobre la compañía, incluyendo el reparto de los derechos de voto y de capital entre los accionistas no familiares, con posibilidad legal o jurídica, y (iii) los miembros de la familia deben tener puestos de alta dirección.

Siguiendo esta propuesta, para la determinación del carácter familiar o no de la empresa, se consideró por un lado en el cuestionario la inclusión de un indicador que permitiese medir si la mayor parte de la propiedad de la empresa se encontraba en manos de la familia, tal y como hicieran algunos trabajos previos (Reyes y Sacristán, 2003). Asimismo, para evaluar la percepción del encuestado en cuanto al control, se incorporó un ítem dicotómico que simplemente cuestionase si el respondiente percibía o no la empresa como familiar. Por último, y de acuerdo con Villalonga y Amit (2004), se solicitó al encuestado que indicase el porcentaje de miembros de la alta dirección que pertenecían a la familia.

Para el análisis de las tres dimensiones indicadas y poder realizar alguna comparación entre las mismas, se ha considerado conveniente dicotomizar aquellas cuestiones evaluadas a través de porcentajes. De esta forma, la evaluación del carácter familiar de las Pymes de la muestra quedaría recogida en la Tabla 2.

Como puede observarse a primera vista a partir de las frecuencias, dos de las tres dimensiones consideradas coincidirían casi a la perfección a la hora de determinar el carácter familiar, mientras que el porcentaje sobre los directivos de la alta dirección estaría indicando que la cantidad de EF es inferior.

\section{Tabla 2}

Distribución de las empresas en función del carácter familiar.

\begin{tabular}{|c|c|c|c|}
\hline \multicolumn{2}{|l|}{ Carácter Familiar } & \multirow{2}{*}{$\begin{array}{l}\text { Frecuencia } \\
169\end{array}$} & \multirow{2}{*}{$\begin{array}{l}\text { Porcentaje } \\
35,14 \%\end{array}$} \\
\hline Percepción de empresa & No & & \\
\hline familiar & Sí & 312 & $64,86 \%$ \\
\hline \multirow{2}{*}{$\begin{array}{l}\text { Propiedad en manos de } \\
\text { la familia }\end{array}$} & $<50 \%$ & 168 & $34,93 \%$ \\
\hline & $\geq 50 \%$ & 313 & $65,07 \%$ \\
\hline Directivos familiares & $<50 \%$ & 195 & $40,54 \%$ \\
\hline ( 1 caso perdido) & $\geq 50 \%$ & 285 & $59,46 \%$ \\
\hline
\end{tabular}

En cualquier caso, a partir de los resultados, podría afirmarse que aproximadamente entre el $60 \mathrm{y}$ el $65 \%$ de las 481 empresas que componen la muestra pudieran ser consideradas como familiares.

Entrando un poco más en detalle en estas tres dimensiones del carácter familiar, aunque al observar el recuento de las dos primeras (percepción y propiedad) pudiera interpretarse que tan sólo en un caso de las 481 empresas no coinciden las respuestas dadas, lo cierto es que son 15 los encuestados que parecen no responder coherentemente a las dos cuestiones. De este modo, de esos 15 casos, 8 afirmaban no percibir el negocio como familiar aún cuando aseguraban que al menos el $50 \%$ de la propiedad de la empresa estaba en manos de la familia. Por el contrario, 7 encuestados percibían el negocio como familiar a pesar de que la familia no tuviera la mayoría de la propiedad en sus manos. Las discrepancias observadas pueden tener su origen en diversos motivos: en primer lugar, a la vista de los resultados, parece ser que los respondientes ven más fácil o sencillo responder preguntas de carácter dicotómico que otras de carácter más abierto en las que se deben aportar datos cuantitativos; además, también debe contemplarse la posibilidad de que el entrevistado no conozca la verdadera distribución de la propiedad de la empresa en la que trabajan, sobre todo cuando el tamaño organizativo aumenta; cabe también la posibilidad de que, si bien el encuestado conoce que la familia posee más del $50 \%$ de la propiedad, el posicionamiento alejado de ésta en la empresa no facilite la difusión en el entorno laboral de las pautas de comportamiento asociadas típicamente al ámbito familiar y que, por ello, no perciban el negocio como tal. 
En el presente estudio, de acuerdo con las recomendaciones de Villalonga y Amit (2004), el carácter familiar de las Pymes de la muestra fue evaluado utilizando como criterio de segmentación el cruce de los tres criterios de estratificación vistos: percepción, propiedad y porcentaje de directivos (Tabla 3).

\section{Tabla 3}

Distribución final de las empresas en función del carácter familiar.

\begin{tabular}{lccc}
\hline \multicolumn{2}{c}{ Carácter Familiar } & Frecuencia & Porcentaje \\
\hline $\begin{array}{l}\text { Cumple los } \\
\text { tres requisitos }\end{array}$ & No & 202 & $42,08 \%$ \\
$\begin{array}{l}\text { (caso } \\
\text { perdido) }\end{array}$ & Sí & 278 & $57,02 \%$ \\
\hline
\end{tabular}

De esta forma, aunque el porcentaje inicialmente previsto se reduciría a poco más del $57 \%$, sí que permite asegurar que las Pymes consideradas como familiares, lo son casi con total seguridad. De cualquier modo, que el $57 \%$ de las Pymes de las 481 empresas de la muestra sean familiares, pone de relieve la importancia que este tipo de estructura organizativa tiene en el tejido empresarial. género del gerente o propietario y a la formación universitaria o no de éste. Asimismo, empleando el estadístico Chi-cuadrado de Pearson por tratarse de variables categóricas se ha contrastado estadísticamente si existe algún tipo de asociación estadística significativa entre las variables. La Tabla 4 recoge las frecuencias obtenidas atendiendo a si le empresa cumple con el carácter familiar o no definido a partir de la agregación de las tres cualidades requeridas: percepción familiar, propiedad familiar y directivos familiares.

A la vista de los resultados puede afirmarse que en términos generales la empresa familiar de la muestra seleccionada es una empresa con menos de 50 empleados, con un gerente propietario varón y sin formación universitaria. Esta generalización coincidiría con la realizada para la muestra en su conjunto, ya que como se desprende del recuento, el número de empresas pequeñas es casi tres veces superior al de medianas, el número de hombres gerentes es casi doce veces superior al de mujeres $\mathrm{y}$, por último, la cantidad de gerentes con estudios universitarios era ligeramente superior al número de gerentes sin estudios universitarios.

De las tres variables analizadas, sólo las que tienen que ver con el gerente, esto es, su género y su

Tabla 4

Características de las empresas familiares y no familiares.

\begin{tabular}{|c|c|c|c|c|c|c|c|c|}
\hline \multirow{3}{*}{ Factores } & & \multicolumn{7}{|c|}{$\begin{array}{l}\text { La empresa puede ser considerada como } \\
\text { Familiar }\end{array}$} \\
\hline & & \multicolumn{2}{|l|}{ No } & \multicolumn{3}{|l|}{ Sí } & \multirow[b]{2}{*}{ Valor $\chi^{2}$} & \multirow[b]{2}{*}{ P-value } \\
\hline & & $\mathrm{N}^{\mathrm{o}}$ & $\%$ & $\mathrm{~N}^{\mathrm{o}}$ & $\%$ & Total & & \\
\hline & & 202 & 42,08 & 278 & 57,92 & 480 & & \\
\hline \multirow{2}{*}{ Tamaño } & Pequeña & 142 & 40,00 & 213 & 60,00 & 355 & \multirow{2}{*}{2,428} & \multirow{2}{*}{,119 } \\
\hline & Mediana & 60 & 45,83 & 65 & 54,17 & 125 & & \\
\hline \multirow{2}{*}{ Género Gerente } & Masculino & 196 & 44,24 & 247 & 55,76 & 443 & \multirow{2}{*}{11,006} & \multirow{2}{*}{, $001 * * *$} \\
\hline & Femenino & 6 & 16,22 & 31 & 83,78 & 37 & & \\
\hline \multirow{2}{*}{$\begin{array}{l}\text { Formación } \\
\text { Gerente }\end{array}$} & $\begin{array}{l}\text { No } \\
\text { universitario }\end{array}$ & 84 & 33,07 & 170 & 66,93 & 254 & \multirow[t]{2}{*}{17,978} & \multirow[t]{2}{*}{, $000 * * *$} \\
\hline & Universitario & 118 & 52,21 & 108 & 47,79 & 226 & & \\
\hline
\end{tabular}

$* * * 99 \%(\mathrm{p}<0,01) ; * * 95 \%(\mathrm{p}<0,05) ; * 90 \%(\mathrm{p}<0,01)$

\section{Discusión de resultados}

Empleando el carácter familiar como un factor de segregación, se ha realizado un análisis descriptivo de la muestra atendiendo al tamaño de la empresa, al formación universitaria, mantienen una asociación estadísticamente significativa con el carácter familiar, tal y como reflejan los resultados del estadístico Chi-cuadrado de Pearson. En función de éste sólo se puede rechazar la hipótesis de 
independencia y concluir, por tanto, que mantienen relación con el carácter familiar, en el caso del género y de la formación del gerente. Así, puede afirmarse que es más probable que una mujer dirija una empresa familiar que no familiar y que es más probable que los gerentes sin cualificación universitaria se encuentren al frente de una empresa familiar que no familiar. El primero de estos encuentros, no hace más que reflejar las dificultades que las mujeres encuentran para poder llegar a liderar organizaciones, siendo más probable que gestionen empresas familiares debido al respaldo que desde su propia familia podrían estar recibiendo. Por otro lado, el segundo hallazgo puede tener diversas interpretaciones, así por ejemplo podría interpretarse que existe una cierta despreocupación por la formación universitaria en aquellos casos en los que el relevo generacional supone una garantía profesional; asimismo también cabe la posibilidad de que precisamente sea la falta de estudios universitarios lo que llevase a muchos de los gestores de las empresas familiares encuestadas a montar su propia empresa.

H1: Las Pymes familiares perciben mayores motivaciones y menores barreras hacia la RSE que las no familiares.

En la siguiente Tabla 5 se muestran las puntuaciones medias obtenidas por sendos grupos de empresas (familiares y no familiares) para cada una de las motivaciones extraídas de la literatura. Por otro lado, tras comprobar previamente que los datos no seguían una distribución normal, se ha realizado

Tabla 5

Asociación de las motivaciones y el carácter familiar.

En qué grado los siguientes aspectos suponen una motivación para implantar/desarrollar RSE en su empresa:

MOT.1 Razones éticas

MOT.2 Los valores sociales y ambientales son más importantes que la rentabilidad

MOT.3 Satisfacción y motivación de los empleados

MOT.4 Satisfacción y fidelización de los clientes

MOT.5 Imagen y reputación que genera para la empresa

MOT.6 Incremento de los beneficios (gracias a la RSE)

MOT.7 Reduce los costes (gracias a la RSE)

MOT.8 Sirve para encauzar el negocio en nuevas líneas relacionadas con lo social y medioambiental

MOT.9 Ayuda a captar clientes con preferencias sociales/medioambientales

MOT.10 Cumplir con la legislación

MOT.11 Mejora las relaciones con sus proveedores

MOT.12 Contribuye a una mayor vinculación con la comunidad

MOT.13 Atención de las demandas de los empleados (presiones internas)

MOT.14 Mejorar la competitividad de la organización

MOT.15 Para obtener ventajas al contratar con las Administraciones Públicas

MOT.16 Ayudas de las Administraciones Públicas

\section{Carácter Familiar}

Media Valores medios

$2,99 \quad 2,98 \quad 3,00 \quad 919$

$3,52 \quad 3,54 \quad 3,50 \quad, 442$

$\underline{3,85} \quad \underline{3,86} \quad \underline{3,84} \quad, 895$

$\underline{3,98}$

$\underline{3,98} \quad \underline{3,98} \quad, 885$

$3,39 \quad 3,35$

$3,27 \quad 3,25$

, 651

$3,36 \quad 3,47$

$3,37 \quad 3,45$

$\underline{3,87} \quad \underline{3,83}$

$3,31 \quad 3,36$

$3,29 \quad 3,41$

3,16

$3,10 \quad 3,20 \quad, 538$

3,52

$3,56 \quad 3,50$

3,43

$3,48 \quad 3,38$

3,37

$3,48 \quad 3,29 \quad, 060 *$

$* * * 99 \%(\mathrm{p}<0,01) ; * * 95 \%(\mathrm{p}<0,05) ; * 90 \%(\mathrm{p}<0,01)$

Herrera, J., Larrán M., Lechuga, M.P. y Martínez, D. (2014). Motivaciones hacia la Responsabilidad Social en las PYMEs familiares. Revista de Empresa Familiar, 4(1), 21-44. 
un contraste de medias utilizando la U de MannWhitne con la intención de analizar ara evaluar si efectivamente las Pymes familiares perciben, por término medio, mayores motivaciones hacia la implementación de prácticas de RSE que las no familiares.

A primera vista y con independencia del carácter familiar, se puede ver cómo la principal motivación es la asociada con la imagen y reputación de las empresas, seguida de cerca por la satisfacción y fidelización de los clientes y por el cumplimiento de la legislación. Las medias entre familiares y no familiares son tan cercanas que no permiten apreciar diferencias significativas entre sendos grupos de empresas. Que estas sean los principales aceleradores viene a poner de manifiesto una mayor empresa en el largo plazo, por encima de la preocupación que en el corto plazo pudiera existir por incrementar los beneficios o reducir los costes. La reputación y la imagen se tornan en ventajas competitivas esenciales en el ámbito de la pequeña y mediana empresa, con independencia del carácter familiar.

Donde sí que el carácter familiar incide de forma significativa es en las motivaciones relacionadas con los valores sociales y ambientales y en la motivación que supone la obtención de ayudas de las administraciones públicas. Respecto a la primera, las EF perciben en mayor medida que los valores sociales y ambientales son más importantes para el desarrollo de prácticas de RSE que la rentabilidad. En cuanto a la segunda, son precisamente las

Tabla 6

Asociación de barreras y carácter familiar

\begin{tabular}{|c|c|c|c|c|c|}
\hline \multirow{3}{*}{\multicolumn{2}{|c|}{$\begin{array}{l}\text { Indique su grado de acuerdo con las siguientes Barreras/Frenos } \\
\text { para implantar/desarrollar RSE en su empresa }\end{array}$}} & \multirow{3}{*}{ Media } & \multicolumn{3}{|c|}{ Carácter familiar } \\
\hline & & & \multicolumn{2}{|c|}{ Valores medios } & \multirow[t]{2}{*}{ P-value } \\
\hline & & & \multirow{2}{*}{$\begin{array}{l}\text { No } \\
3,35\end{array}$} & Sí & \\
\hline BAR.1 & Falta de formación sobre la RSE & 3,36 & & 3,37 & ,936 \\
\hline BAR.2 & Falta de información adecuada & $\underline{3,43}$ & $\underline{3,41}$ & $\underline{3,44}$ & ,952 \\
\hline BAR.3 & Escasez de recursos financieros & $\underline{3,42}$ & $\underline{3,46}$ & $\underline{3,39}$ & ,588 \\
\hline BAR.4 & Escasez de recursos humanos & 3,22 & 3,31 & 3,16 &, 161 \\
\hline BAR.5 & Falta de tiempo & $\underline{3,55}$ & $\underline{3,47}$ & $\underline{3,61}$ & ,337 \\
\hline BAR.6 & Estas prácticas no mejoran el resultado económico & 3,09 & 3,12 & 3,07 & ,620 \\
\hline BAR.7 & $\begin{array}{l}\text { La legislación medioambiental no es relevante para } \\
\text { mi negocio }\end{array}$ & 2,89 & 2,91 & 2,87 & 601 \\
\hline BAR. 8 & $\begin{array}{l}\text { Mis proveedores no consideran importantes las } \\
\text { cuestiones sociales medioambientales }\end{array}$ & 2,91 & 2,90 & 2,92 & ,923 \\
\hline BAR.9 & $\begin{array}{l}\text { Mis clientes no consideran importantes las cuestiones } \\
\text { sociales medioambientales }\end{array}$ & 2,98 & 2,97 & 2,99 & ,804 \\
\hline BAR.10 & $\begin{array}{l}\text { Mis empleados no consideran importantes las } \\
\text { cuestiones sociales medioambientales }\end{array}$ & 2,81 & 2,76 & 2,85 & ,389 \\
\hline BAR.11 & $\begin{array}{l}\text { La dificultad de integrar los aspectos sociales y } \\
\text { ambientales en la estrategia de la empresa }\end{array}$ & 3,23 & 3,12 & 3,31 &, $047 * *$ \\
\hline BAR.12 & $\begin{array}{l}\text { La falta de servicios adecuados de apoyo para las } \\
\text { Pymes }\end{array}$ & $\underline{3,80}$ & $\underline{3,71}$ & $\underline{3,86}$ & ,088* \\
\hline BAR.13 & Baja competitividad de la economía local & 3,26 & 3,20 & 3,31 & 270 \\
\hline
\end{tabular}

Nota: $* * * 99 \%(\mathrm{p}<0,01) ; * * 95 \%(\mathrm{p}<0,05) ; * 90 \%(\mathrm{p}<0,01)$

preocupación por encontrar soluciones estratégicas que garanticen la viabilidad y la sostenibilidad de la empresas familiares las que otorgan una puntuación inferior a la ventaja que la RSE puede suponer para 
la obtención de determinadas ayudas públicas. Sendos hallazgos estarían poniendo de manifiesto que en el caso de las empresas de carácter familiar existe un predominio del interés normativo por la RSE (más relacionado con la ética, la moral y los valores) sobre el carácter instrumental de ésta (más cercano a la racionalidad económica y competitiva).

Igualmente, la Tabla 6 recoge un análisis similar pero en este caso, considerando las principales barreras o frenos que los encuestados de las Pymes percibían de cara a la implementación de la RSE en sus empresas.

En esta ocasión, la principal barrera que con independencia del carácter familiar ven los respondientes es la falta de servicios de apoyo para las Pymes, lo cual pone en evidencia que aunque las Pymes conforman la mayor parte del tejido productivo empresarial, la mayoría de normativas, guías y documentos de apoyo elaborados hasta el momento tiene como objeto de aplicación a las grandes corporaciones. La propia estructura organizativa de la mayoría de las Pymes encuestadas (recuérdese que el 73,80\% eran pequeñas empresas) provoca que ante la falta de información existente en cuanto a qué es la RSE y los escasos recursos financieros con los que cuentan actualmente las Pymes, el tiempo disponible para poder actualizar conocimientos e implementar una gestión sostenible sea otro de los principales inconvenientes.

El carácter familiar sólo tiene una incidencia significativa a la hora de puntuar como barreras la dificultad de integrar los aspectos sociales y ambientales en la estrategia de la empresa y la falta de servicios adecuados de apoyo a las Pymes, siendo las EF las que otorgan una mayor puntuación en ambos casos y, por tanto, las que perciben mayores barreras.

Las discrepancias observadas en relación a los aceleradores y barreras que por regla general perciben las Pymes familiares y no familiares no permiten soportar la hipótesis uno en sentido amplio. Si bien, del estudio pormenorizado entre familiares y no familiares sólo puede hacerse hincapié en el hecho de que las EF parecen presentar una orientación menos preocupada por la repercusión que sobre los resultados económicos pudiera derivarse del comportamiento socialmente responsable y que, en cambio, muestran un mayor interés por actuar conforme a la ética y valores sociales y ambientales que imperan en las mismas. Por otro lado, que sean las EF las que perciben en mayor medida las barreras señaladas, podría estar poniendo de manifiesto que este tipo de entidades muestran una mayor preocupación por implementar e integrar en su estrategia la RSE y que, por ello, tienen un mayor conocimiento de las dificultades que supone la falta de apoyo para integrar las cuestiones sociales y ambientales.

Una vez analizadas las diferentes percepciones entre familiares y no familiares, se ha centrado la atención en aquellas consideradas como familiares. De esta forma, se ha examinado cómo el género del gerente, su formación y el tamaño de las $278 \mathrm{EF}$ de la muestra pueden mantener algún tipo de relación con las motivaciones y barreras que este tipo de organizaciones perciben de cara a la implementación de RSE en sus empresas (Tabla 7).

\section{Tabla 7}

Análisis descriptivo de la composición de empresas familiares.

\begin{tabular}{llll}
\hline Género & Formación & $\begin{array}{l}\text { Tamaño } \\
\text { Pequeña }\end{array}$ & Mediana \\
& $\begin{array}{l}\text { Gerente No } \\
\text { Universitario }\end{array}$ & 125 & 29 \\
$\begin{array}{l}\text { Gerente } \\
\text { Hombre }\end{array}$ & Gerente & & \\
& Universitario & 62 & 31 \\
& $\begin{array}{l}\text { Gerente No } \\
\text { Universitario }\end{array}$ & 15 & 1 \\
Gerente & Gerente & 11 & 4 \\
Mujer & Universitario & & \\
& &
\end{tabular}

Para ello, antes de comenzar con el contraste de las hipótesis planteadas, se ha elaborado una tabla de contingencias que permitiese identificar frecuencias. Asimismo, dado que las características consideradas son variables categóricas, se analizó la correlación por pares entre las mismas a partir de la Chicuadrado de Pearson, encontrando únicamente una asociación significativa al $95 \%$ de confianza $(\chi 2=$ 8,032 ) entre el tamaño y la formación del gerente. De forma que los gerentes sin formación universitaria tienden a encontrarse al frente de empresas de reducida dimensión.

A continuación, una vez conocida la composición de la muestra de EF objeto del estudio, se ha llevado a cabo el contraste de las tres hipótesis restantes. Para ello, las Tablas 8 y 9 recogen las puntuaciones medias y los valores de significación asintótica bilateral resultantes al aplicar la prueba $U$ de Mann-Whitney para el contraste de las hipótesis: 
H2: Las Pymes familiares que cuentan con un propietario gerente mujer perciben mayores motivaciones y menores barreras que las que tienen un propietario gerente hombre.

H3: Las Pymes familiares que cuentan con un propietario gerente con cualificación universitaria perciben mayores motivaciones y menores barreras que las que tienen un propietario gerente sin estudios universitarios.

H4: Las empresas familiares medianas perciben mayores motivaciones y menores barreras que las pequeñas.

Por orden, al observar las puntuaciones medias atendiendo al género de gerente puede verse que salvo en dos casos (Mot. 8 y Mot. 10), las Pymes familiares que tienen por gerente a una mujer perciben en mayor medida las motivaciones que aquellas en las que la figura del gerente o máximo responsable la desempeña un hombre. No obstante, esta diferencia es únicamente significativa desde un punto de vista estadístico cuando se pregunta al encuestado por la incidencia que la obtención de ventajas al contratar con las administraciones públicas puede tener sobre el desarrollo de prácticas sostenibles. Este hecho podría ser debido a que desde las administraciones públicas se presta, desde hace tiempo, especial atención a la capacidad emprendedora de las mujeres. Existen múltiples programas de seguimiento y planificación estratégica orientados hacia la mujer, de modo que ellas pueden percibir en mayor medida las potenciales ventajas de contratación con las administraciones públicas que sus homólogos masculinos.

Prestando atención a la formación universitaria del gerente se observa que, de nuevo para casi todas las motivaciones a excepción de la séptima y la duodécima, las Pymes familiares con un gerente universitario tienden a otorgar puntuaciones más elevadas a las motivaciones que los gerentes sin formación universitaria. En esta ocasión son tres las motivaciones en las que estas diferencias son significativas al $95 \%$ de confianza. Así, puede rechazarse la hipótesis nula de independencia entre Mot.1, 10 y 16 y la variable formación universitaria. Estos resultados corroboran que los gerentes con estudios superiores consideran que las razones éticas tienen un mayor peso de cara a la implementación de RSE en las empresas que los que no cuentan con estudios universitarios. Asimismo, derivado quizás del grado de conocimiento adquirido durante sus estudios superiores, consideran también en mayor medida que cumplir con la legislación es una de las principales motivaciones. Por último, esa formación universitaria también guarda una relación significativa y positiva con la relevancia que tienen las ayudas que las administraciones públicas ofertan a aquellas empresas que implementen prácticas de RSE.

La última de las características organizativas analizadas ha sido el tamaño. A primera vista, las empresas medianas puntúan doce de los dieciséis aceleradores por encima de la puntuación otorgada por las pequeñas. El contraste de medias permite afirmar que, conforme a lo esperado, las pequeñas empresas familiares muestran un mayor interés por la repercusión que la RSE puede tener a la hora de reducir costes y, aunque no de forma significativa, por aumentar los ingresos. Por otro lado los resultados obtenidos respecto a la incidencia que las razones éticas y los valores sociales y ambientales tienen para el desarrollo de prácticas sostenibles resultan llamativos. Si bien a priori cabría esperar una relación inversa entre el tamaño y la valoración otorgada a estas motivaciones, los resultados evidencian lo contrario $\mathrm{y}$, además, de forma significativa. Se demuestra, por tanto, que las empresas familiares medianas otorgan una mayor importancia a las razones éticas y a los valores sociales y ambientales que las pequeñas, más preocupadas por el potencial que tiene la RSE de mejorar su rentabilidad y su competitividad.

Al realizar el contraste observando las barreras, prácticamente no se aprecian diferencias significativas en función de las características analizadas. Por un lado, en cuanto al género y la formación ya no parece existir ninguna tendencia que permita diferenciar a las mujeres gerentes de los hombres y a los que tienen estudios universitarios de los que no. Sí que al atender a las puntuaciones medias otorgadas por pequeñas y medianas se observa que, salvo en dos barreras (Bar. 4 y Bar.8), las empresas familiares pequeñas tienden a percibir más dificultades que las medianas para implementar prácticas de RSE. No obstante, ninguna de estas diferencias en cuanto a tamaño es estadísticamente significativa. 
Tabla 8

Asociación entre las motivaciones de la RSE y algunas características de las empresas familiares

\begin{tabular}{|c|c|c|c|c|c|c|c|c|c|c|}
\hline \multirow{4}{*}{ MOT. } & \multirow{4}{*}{ Media } & \multicolumn{3}{|c|}{$\begin{array}{l}\text { GERENTE } \\
\text { MUJER }\end{array}$} & \multicolumn{3}{|c|}{$\begin{array}{c}\text { GERENTE } \\
\text { UNIVERSITARIO }\end{array}$} & \multicolumn{3}{|c|}{ EMPRESA MEDIANA } \\
\hline & & \multirow{2}{*}{\multicolumn{2}{|c|}{ Valores medios }} & \multirow{3}{*}{ P-value } & \multirow{2}{*}{\multicolumn{2}{|c|}{$\begin{array}{l}\text { Valores } \\
\text { medios }\end{array}$}} & \multirow{3}{*}{ P-value } & \multirow{2}{*}{\multicolumn{2}{|c|}{$\begin{array}{l}\text { Valores } \\
\text { medios }\end{array}$}} & \multirow{3}{*}{ P-value } \\
\hline & & & & & & & & & & \\
\hline & & No & Sí & & No & Sí & & No & Sí & \\
\hline MOT.1 & 3,00 & 3,00 & 3,05 & 890 & 2,91 & 3,16 &, $044 * *$ & 2,94 & 3,23 &, $060^{*}$ \\
\hline MOT.2 & 3,09 & 2,97 & 3,08 &, 720 & 3,02 & 3,19 &, 112 & 3,01 & 3,34 &, $014 * *$ \\
\hline MOT.3 & 3,50 & 3,50 & 3,59 & ,687 & 3,43 & 3,59 &, 127 & 3,46 & 3,58 & ,255 \\
\hline MOT.4 & 3,84 & 3,83 & 3,97 & ,266 & 3,77 & 3,94 & ,166 & 3,81 & 3,92 & ,444 \\
\hline MOT.5 & 3,98 & 3,95 & 4,11 &, 117 & 3,91 & 4,06 &, 177 & 3,95 & 4,03 &, 752 \\
\hline MOT.6 & 3,35 & 3,35 & 3,59 &, 111 & 3,32 & 3,43 & ,273 & 3,40 & 3,23 &, 365 \\
\hline MOT.7 & 3,25 & 3,23 & 3,32 & ,917 & 3,24 & 3,22 & 894 & 3,29 & 3,03 &, $089^{*}$ \\
\hline MOT.8 & 3,47 & 3,42 & 3,38 &, 506 & 3,42 & 3,52 &, 511 & 3,46 & 3,47 &, 726 \\
\hline MOT.9 & 3,45 & 3,41 & 3,43 & 879 & 3,37 & 3,54 & ,228 & 3,42 & 3,48 &, 861 \\
\hline MOT.10 & 3,83 & 3,85 & 3,78 & 619 & 3,75 & 4,01 &, $037 * *$ & 3,79 & 4,03 &, 131 \\
\hline MOT.11 & 3,36 & 3,32 & 3,38 &, 538 & 3,27 & 3,45 &, 121 & 3,32 & 3,40 & ,466 \\
\hline MOT.12 & 3,41 & 3,34 & 3,43 & ,466 & 3,4 & 3,39 & ,976 & 3,38 & 3,45 & ,624 \\
\hline MOT.13 & 3,20 & 3,14 & 3,32 & ,446 & 3,12 & 3,31 &, 114 & 3,17 & 3,28 &, 405 \\
\hline MOT.14 & 3,50 & 3,50 & 3,57 & ,301 & 3,41 & 3,60 &, 130 & 3,50 & 3,43 & ,788 \\
\hline MOT.15 & 3,38 & 3,39 & 3,65 & ,064* & 3,29 & 3,50 &, 154 & 3,40 & 3,27 &, 563 \\
\hline MOT.16 & 3,29 & 3,34 & 3,46 & ,430 & 3,14 & 3,45 &, $046 * *$ & 3,25 & 3,29 & ,703 \\
\hline
\end{tabular}

Nota: ***99\% $(\mathrm{p}<0,01) ; * * 95 \%(\mathrm{p}<0,05) ; * 90 \%(\mathrm{p}<0,01)$

$\mathrm{Si}$ nos detenemos en cada una de las características, aquellas EF dirigidas por una gerente perciben en mayor medida que la inexistencia de una legislación medioambiental aplicable a sus negocios incide negativamente en la incorporación estratégica de la RSE. Dicho de otro modo, son las empresas gestionadas por mujeres las que afirman que, de no existir legislación medioambiental aplicable, mostrarían un menor interés por la RSE. Este hecho, deja entre ver que este tipo de EF tienden más a una implementación estratégica de la RSE reactiva que proactiva.

Por otro lado, las EF gestionadas por un gerente masculino, otorgan una mayor importancia a las dificultades derivadas de la integración de los aspectos sociales y ambientales en la estrategia de la empresa, resultado que pudiera explicarse atendiendo al porcentaje de gerentes masculinos con estudios universitarios. Así, que más del $60 \%$ de los gerentes hombres no tengan formación universitaria pudiera ser la causa de que, por término medio, los propietarios gerentes hombres encuentren mayores dificultades para integrar estratégicamente determinadas cuestiones sostenibles.

En último lugar, en relación con el género, es de destacar la diferencia encontrada en las puntuaciones medias que tienen que ver con los recursos financieros (Bar. 3), a pesar de no ser estadísticamente significativa. Las EF dirigidas por mujeres no perciben al igual que los hombres que la escasez de recursos financieros sea una posible justificación de la no implementación de prácticas sostenibles. Sin embargo, las Pymes dirigidas por hombres sí que lo consideran un freno relevante, de hecho, si se atiende a las puntuaciones medias, las dirigidas por hombres lo califican como el tercer 
Tabla 9

Asociación entre las barreras de la RSE y algunas características de las empresas familiares.

\begin{tabular}{|c|c|c|c|c|c|c|c|c|c|c|}
\hline \multirow[t]{2}{*}{ BAR. } & \multirow[t]{2}{*}{ Media } & & $\begin{array}{l}\text { erent } \\
\text { ores } \\
\text { lios }\end{array}$ & P-value & & $\begin{array}{l}\text { nte Un } \\
\text { ores } \\
\text { lios }\end{array}$ & rsitario & & $\begin{array}{l}\text { presa } \\
\text { ores } \\
\text { lios }\end{array}$ & diana \\
\hline & & No & Sí & & No & Sí & & No & Sí & \\
\hline BAR.1 & 3,37 & 3,35 & 3,32 & ,473 & 3,43 & 3,26 &, 186 & 3,42 & 3,18 &, 164 \\
\hline BAR.2 & 3,44 & 3,42 & 3,43 & ,954 & 3,51 & 3,32 & , 180 & 3,49 & 3,25 & , 193 \\
\hline BAR.3 & 3,39 & 3,45 & 3,14 &, 122 & 3,52 & 3,22 &, $047 * *$ & 3,45 & 3,26 & 290 \\
\hline BAR.4 & 3,16 & 3,24 & 3,16 & ,653 & 3,21 & 3,09 &, 372 & 3,15 & 3,23 & ,553 \\
\hline BAR.5 & 3,61 & 3,58 & 3,49 & ,339 & 3,66 & 3,57 & ,471 & 3,64 & 3,58 & ,499 \\
\hline BAR.6 & 3,07 & 3,06 & 3,32 &, 150 & 3,04 & 3,08 &, 701 & 3,08 & 2,97 & ,410 \\
\hline BAR.7 & 2,87 & 2,85 & 3,22 &, $072 *$ & 2,81 & 2,93 & ,456 & 2,86 & 2,85 & ,832 \\
\hline BAR. 8 & 2,92 & 2,91 & 2,89 &, 621 & 2,85 & 3,03 & ,201 & 2,89 & 3,03 & ,333 \\
\hline BAR.9 & 2,99 & 2,97 & 3,00 &, 875 & 2,92 & 3,09 & ,186 & 3,04 & 2,80 & ,107 \\
\hline BAR.10 & 2,85 & 2,79 & 3,08 &, 144 & 2,77 & 2,97 &, 154 & 2,87 & 2,80 & ,460 \\
\hline BAR.11 & 3,31 & 3,25 & 3,05 &, $058 *$ & 3,28 & 3,37 &, 447 & 3,32 & 3,31 &, 851 \\
\hline BAR.12 & 3,86 & 3,80 & 3,70 &, 525 & 3,83 & 3,89 &, 819 & 3,9 & 3,71 & ,238 \\
\hline BAR.13 & 3,31 & 3,26 & 3,32 & ,917 & 3,37 & 3,19 & ,255 & 3,33 & 3,22 &, 767 \\
\hline
\end{tabular}

freno más importante mientras que para el género femenino es el noveno.

En cuanto a la percepción de las barreras en función de la formación universitaria o no del gerente, tan solo se ha observado una diferencia significativa, la relacionada con la escasez de recursos financieros (Bar. 3). De este modo, para los gerentes sin formación universitaria las limitaciones de recursos financieros son más importantes que para los gerentes universitarios. La ausencia de estudios superiores no permite a los gerentes tener una visión amplia de los requisitos que realmente debe aunar una adecuada implementación de la RSE, reduciendo su campo de visión a lo estrictamente financiero.

La formación universitaria también incide, aunque no de forma significativa, sobre la valoración que los encuestados hacen de las barreras que tienen que ver con los proveedores, los clientes y los empleados. De este modo, aquellos gerentes que fueron a la universidad perciben en mayor medida la relevancia que estos stakeholders pueden llegar a tener como factor de presión de cara a la implementación de prácticas sostenibles. Asimismo, y de nuevo no significativamente, resulta coherente que sean los gerentes sin estudios universitarios los que atribuyan, en mayor medida, la falta de desarrollo de prácticas sostenibles a la escasa formación e información que tienen de la RSE.

Ya por último, y como se ha comentado, el tamaño que tienen las Pymes familiares de la muestra no incide significativamente sobre ninguna de las percepciones acerca de las barreras o frenos. No obstante, las pequeñas perecen otorgar más importancia a los frenos que las medianas. Especialmente se encuentran diferencias absolutas entre medias en cuanto a las barreras 1, 2 y 3. Las dos primeras, relacionadas con la formación y la información, seguramente se deban a que 140 de las 213 pequeñas empresas familiares que se analizaron tienen como gerente a una persona sin cualificación universitaria.

Por otro lado, en cuanto a la importancia otorgada por las pequeñas a la ausencia de importancia que los clientes otorgan a la RSE, su explicación podría radicar en que por regla general este tipo de empresas tienden a mantener un contacto más cercano y directo con el clienteconsumidor del producto final, el cual, en muchos 
casos desconoce qué es la RSE y qué se persigue fomentando este tipo de prácticas.

Las medianas, en cambio, probablemente desarrollan su actividad en un ámbito comercial distinto, en el sentido de que en muchas ocasiones estas empresas actúan, a su vez, como proveedoras de entidades de mayor tamaño que sí que conocen la RSE y que exigen, entre otras cuestiones, el cumplimiento de determinados requerimientos de carácter ambiental y social.

\section{Conclusiones}

Es indiscutible que la RSE se ha convertido en objeto de atención por parte de académicos y profesionales. $\mathrm{Su}$ conceptualización como comportamiento y su incidencia en el ámbito económico, social y medioambiental de las grandes corporaciones han acaparado la mayor parte de esa atención hasta el momento. Si bien, las conclusiones derivadas del estudio de este fenómeno no pueden extrapolarse, sin más, al ámbito de las Pymes y menos aún, al de las Pymes familiares.

Tras una primera revisión de la literatura previa sobre las motivaciones y barreras que perciben este tipo de organizaciones, puede afirmarse que las razones que justifican su comportamiento sostenible son similares a las señaladas por grandes corporaciones y que tienen que ver con la reputación y la imagen corporativa, con la ética y los valores sociales y ambientales del gerente o directivo, con el rendimiento financiero y competitivo, y con la presión de los diferentes stakeholders. En este sentido, este trabajo corrobora que si bien desde las Pymes se continúa percibiendo cierta preocupación por la incidencia que sobre su rendimiento a corto plazo puede tener cualquier cambio estratégico, se comienza a apreciar un cierto interés por la supervivencia más allá de los rendimientos inmediatos. Así, al igual que corroborasen trabajos previos (Borga et al., 2009; Nielsen y Thomsen, 2009; Herrera et al., 2013), la preocupación por ofrecer una buena imagen y no empañar la reputación con posibles sanciones se anteponen a los beneficios que la RSE puede generar a través de la reducción de costes o el aumento de los ingresos. Aunque para ello, y como ya apuntasen algunos autores (Halila, 2007; Spence y Perrini, 2009), deban fortalecerse los servicios de apoyo a la integración estratégica de la RSE a disposición de las Pymes.

En relación al carácter familiar, las motivaciones $\mathrm{y}$ barreras que perciben empresas familiares y no familiares no difieren significativamente. Sin haber llegado a comprobar si existen diferencias significativas en cuanto al nivel de desempeño sostenible real de las empresas familiares y no familiares, sí que puede concluirse a priori que no existen importantes diferencias significativas en las razones que justifican el nivel de prácticas implementado por cada una de ellas. Aunque, en términos generales, los resultados obtenidos no coinciden con la mayoría de las evidencias halladas en torno al carácter familiar, sí que pueden esgrimirse determinadas conclusiones a partir de las escasas diferencias significativas encontradas. Así, puede afirmarse que las EF muestran una preocupación significativamente superior por las cuestiones sociales y ambientales, tal y como confirmaron respectivamente Deniz y Suárez (2005) y Walls et al. (2007). Las EF ven que de cara a la implementación de RSE es más importante la capacidad de integrar los valores sociales y ambientales en la estrategia que la obtención de una mejora en la rentabilidad. Asimismo, no les preocupa tanto la obtención de ayudas por parte de las administraciones públicas como el poder contar con servicios de apoyo especializados en el ámbito de las Pymes que les oriente y les facilite la integración estratégica de esos aspectos sociales y ambientales; esta preocupación por la integración estratégica viene a confirmar en cierto modo las afirmaciones de Bingham et al. (2011) en torno a la relevancia que para las EF tiene la visión a largo plazo y la preocupación por un relevo generacional sustentado en ideales. Por otro lado, el estudio empírico desarrollado exclusivamente a partir de EF confirma que la sensibilidad femenina y el nivel de formación del gerente favorecen la implementación de RSE y que son las empresas de menor dimensión las que más dificultades perciben a la hora de ejecutar esa implementación. No obstante, estas afirmaciones no cuentan con el suficiente sustento estadístico y por ello deben ser tomadas con precaución.

Las evidencias encontradas sí que han permitido contrastar cómo las mujeres gerentes al frente de una empresa familiar tienen un mayor conocimiento de las oportunidades contractuales que la RSE ofrece, gracias sobretodo a que es relativamente superior el porcentaje de mujeres gerentes con cualificación universitaria (15 de 31) que de hombres (93 de 247). Por otra parte, aquellas empresas cuyo gerente posee estudios universitarios ofrecen, en cierto modo, una percepción diferenciada y un tanto contradictoria en lo que a los aceleradores y barreras se refiere: por un lado ven la importancia de la ética e identifican que

Herrera, J., Larrán M., Lechuga, M.P. y Martínez, D. (2014). Motivaciones hacia la Responsabilidad Social en las PYMEs familiares. Revista de Empresa Familiar, 4(1), 21-44. 
un comportamiento sostenible no requiere exclusivamente de recursos financieros $y, \sin$ embargo por otro lado, muestran una mayor preocupación por los posibles incumplimientos legales con las respectivas sanciones y la oportunidad que la RSE supone para acceder a determinadas ayudas y subvenciones de carácter público.

En cuanto al tamaño, sorprende que en contra de la mayoría de evidencias previas las empresas medianas puntuasen en mayor medida la capacidad que tienen las razones éticas y los valores sociales y ambientales para promover un comportamiento sostenible. La difusión que la RSE está protagonizando en los últimos años puede haber provocado, que por una cuestión de imagen $y$ reputación, sean las empresas medianas las que en un primer momento se hayan interesado por conocer qué comprende e implica un comportamiento socialmente responsable.

Para finalizar, entendemos que este trabajo no está exento de posibles limitaciones asociadas por ejemplo a la composición de la muestra. Así, ha de tenerse presente que únicamente se contemplaron empresas de una misma región española y que todas les empresas analizadas contaban con entre 10 y 250 empleados, excluyéndose tanto a las microempresas familiares como a las empresas familiares calificadas como grandes corporaciones. En este sentido, la inclusión de sendos grupos de entidades quizás arrojase una mayor luz en lo que a diferencias significativas respecto al tamaño se refiere. Por otro lado, trabajos futuros pueden ser replicados ampliando el estudio a otras comunidades autónomas, permitiendo desarrollar análisis comparativos y contrastar si determinados factores culturales inciden sobre los resultados. Además consideramos que sería de interés en qué medida otras características organizativas como el sector, el tiempo que lleva el negocio en funcionamiento o el número de generaciones que han estado al frente de la empresa inciden sobre las motivaciones y barreras hacia la RSE.

Por último, el trabajo presentado intenta evaluar la predisposición de las Pymes familiares hacia la RSE, esto es motivaciones y barreras. En ningún caso constituye una realidad del desempeño que en materia de sostenibilidad llevan a cabo este tipo de entidades, de ahí el interés que pudiera tener profundizar en el análisis causal del efecto que sobre los verdaderos niveles de implementación en RSE pudieran tener las motivaciones y barreras recogidos en este estudio.

\section{Bibliografía}

Abdellatif, M., Amann, B. y Jaussaud, J. (2010). Family versus non-family business: A comparison of international strategies. Journal of Family Business Strategy, 1(2): 108-116.

Allouche, J., Amann, B. y Kurash, T. (2008). The impact of family control on the performance and financial characteristics of family versus non-family businesses in Japan: A matched-pair investigation. Family Business Review, 21(4), 315-329.

Amann, B, Jaussaudb, J. y Martineza, I (2011). Corporate social responsibility in Japan: Family and nonfamily business differences and determinants. Asian Business \& Management, 11 (3), 329-345.

Astrachan, J. H., Zahra, S. A. y Sharma, P. (2003). Family-sponsored ventures. Kansas, MO: Kauffman Foundation.

Baden, D. A., Harwood, I. A. y Woodward, D.G. (2009). The effect of buyer pressure on suppliers in SMEs to demonstrate CSR practices: an added incentive or counter productive?. European Management Journal. 27,429-441.

Bajo, N. y Duran, J.J. (2009). Responsabilidad social y variables estratégicas en las grandes empresas españolas. Boletín Revista de Responsabilidad Social de la Empresa, $n^{\circ} 2$.

Battaglia, M., Bianchi, L., Frey, M. e Iraldo, F. (2010). An innovative model to promote CSR among SMEs operating in industrial clusters: evidence from an EU project. Corporate Social Responsibility and Environmental Management. 17, pp. 133-141.

Besser, T.L., 1998. The significance of community to business social responsibility. Rural Sociology, 63(3), $412-431$.

Bingham, J. B., Dyer Jr., W. G., Smith, I. y Adams, G. L. (2010). A stakeholder identity orientation approach to corporate social performance in family firms. Journal of Business Ethics, 99(4), 565-585.

Block, J. (2010). Family management, family ownership, and downsizing: evidence from S\&P 500 firms. Family Business Review, 23(2), 1-22.

Borga, F., Citterio, A., Noci, G. y Pizzurno, E. (2009). Sustainability report in small enterprises: case studies in Italian furniture companies. Business Strategy and the Environment. 19, 162-176.

Bos-Brouwers, H. E. J. (2010). Corporate sustainability and innovation in SMEs: evidence of themes and activities in practice. Business Strategy and the Environment. 19, 417-435

Bradford, J. y Fraser, E.D.G. (2008). Local authorities, climate change and small and medium enterprises: identifying effective policy instruments to reduce energy 
use and carbon emissions. Corporate Social Responsibility and Environmental Management. 15, 156172.

Brice, W.D (2013). Family firm culture and performance: Specific empirical evidence. International Journal of Academic Research in Accounting, Finance and Management Sciences, 3 (1), 5-17.

Burgess, Z. y Tharenou, P. (2002). Women Board Directors: Characteristics of the Few. Journal of Business Ethics, 37 (1), 39-49.

Cabrera, M.K, Déniz, M.C. y Santana, D.J. (2005). Responsabilidad Social Corporativa y Empresa Familiar. Revista Europea de Dirección y Economía de la Empresa, 14 (4), 43-58.

Cambell, J.L. (2007). Why Would Corporations Behave In Institutional Theory of Corporate Social Responsibility. Academy of Management Review. 32, (3), 946-967.

Casado, F y Rodríguez, M.A. (2009). La RSE en la gran empresa familiar española. Instituto de la Empresa Familiar. Documento 155. Se puede consultar en: http://www.acefam.org/wp-content/uploads/La-RSE-enla-Empresa-Familiar.pdf (última consulta realizada el 09/04/2014)

Child, J. (1972). Organizational structure, environment and performance: The role of strategic choice. Sociology, 6, 1-22.

Ciliberti, F., Pontrandolfo, P. y Scozzi, B. (2008). Investigating corporate social responsibility in supply chains: a SMEs perspective. Journal of Cleaner Production, 16, 1579-1588.

Ciliberti, F., De Groot, G., De Haan, J. y Pontrandolfo, P. (2009). Codes to coordinate supply chains: SMES' experiences with SA8000. Supply Chain Management: An International Journal. 14 (2), 117-127.

Davies, I. A. y Crane, A. (2010). Corporate Social Responsibility in small and medium size enterprises: investigating employee engagement in fair trade companies. Business Ethics: A European Review. 19 (2), 126-139.

De Vaus, D.A. (1993): "Surveys in Social Research", UCL Press, Londres.

Dawson, S., Breen, J. y Satyen, L. (2002). The ethical outlook of micro business operators. Journal of Small Business Management, 40 (4), 302-313.

De Jong, P., Lancaster, J., Pelaez, P. y Muñoz, J.S. (2008). Examination of correlates of ethical propensity and ethical intentions in the United States, Australia and the Philippines: A managerial perspective. International Journal of Management, 25 (2), 270-278.

Deniz, D. y Suarez, M.K.C. (2005). Corporate social responsibility and family business in Spain. Journal of Business Ethics, 56 (1), 27-36.
De Miguel, M.L. (2011). La Responsabilidad Social Empresarial en la pequeña y mediana empresa: Guía de buenas prácticas. Proyecto Impulsa-RSE Pyme financiado por el Ministerio de Industria, Turismo y Comercio.

Directorio Central De Empresas (DIRCE, 2009) Instituto Nacional De Estadística. Disponible en: http://estadisticas.ipyme.org/InformesEstadisticos/Retrato PYME2009.pdf (última consulta se realiza 09/04/2014).

Dyer, W. G. Jr. y Whetten, D. A. (2006). Family firms and social responsibility: preliminary evidence from the S\&P 500. Entrepreneurship: Theory and Practice, 28, 391-409.

Eilbirt, H. y Parket, I. R. (1973). The current status of corporate social responsibility. Business Horizons, 16 (4), $5-14$.

Fassin, Y. (2008). SMEs and the fallacy of formalising CSR. Business Ethics: A European Review, 17 (4), 364-378.

Fisher, K., Geenen, J., Jurcevic,M., Mcclintock, K. y DaviS, G. (2009). Applying asset-based community development as a strategy for CSR: A Canadian perspective on a win-win for stakeholders and SMEs. Business Ethics: A European Review, 18 (1), 66-82.

Fitzgerald, M., Haynes, G., Schrank, H. y Danes, S. (2010). Socially responsible processes of small family business owners: Exploratory evidence from the national family business survey. Journal of Small Business Management, 48 (4), 524-551.

Fritzsche, D. y Oz, E. (2007). Personal values influence on the ethical dimension of decision making. Journal of Business Ethics, 75 (4), 335-343.

Friedman, A., Miles, S. y Adams, C. (2000). Small and medium-sized enterprises and the environment: evaluation of a specific initiative aimed at all small and medium-sized enterprises. Journal of Small Business and Enterprise Development, 7 (4), 325-342.

Fox, T. (2005). Small and Medium Sized Enterprises (SMEs) and Corporate Social Responsibility: A Discussion Paper, IIED.

Fuller, T. y Tian, Y. (2006). Social and symbolic capital and responsible entrepreneurship: an empirical investigation of SME narratives. Journal of Business Ethics, 67, 287-304.

Gadenne, D., Kennedy, J. y Mckeiver, C. (2009). An empirical study of environmental awareness and practices in SMEs. Journal of Business Ethics, 84, 45-63.

García-Borbolla, A., Herrera Madueño, J. y Larrán Jorge, M (2009). Análisis empírico de la influencia de la propiedad familiar sobre la orientación estratégica de las pequeñas y medianas empresas. Investigaciones Europeas de Dirección y Economía de la Empresa, 15 (1), 45-58.

Godfrey, J. (1995). What's good for women is good for the country. Vital Speeches of the Day, 61(173), 538541. 
Graafland, J.J. (2002). Corporate social responsibility and family business. Paper presented at the Research Forum of the Family Business Network 13 the Annual Conference, Helsinki, Finland.

Graafland J.; Van De Ven B. y Stoffele N. (2003). Strategies and instruments for organising CSR by small and large businesses in the Netherlands. Journal of Business Ethics, 47 (1), 5-60.

Guibert, J. M. (coord.) (2009): Responsabilidad Social Empresarial: Competitividad y casos de buenas prácticas en PYMES, Publicaciones de la Universidad de Deusto, San Sebastián.

Gelbmann, U. (2010). Establishing strategic CSR in SMEs: an Austrian CSR quality seal to substantiate the strategic CSR performance. Sustainable Development, 18 (2), 90-98.

Godos-Díez, J. L., Fernández-Gago, R. y MartínezCampillo, A. (2011). How important are CEOs to CSR practices?: An analysis of the mediating effect of the perceived role of ethics and social responsibility. Journal of Business Ethics, 98 (4), 531-548.

Granitz, N.A., (2003). Individual, social y organizational sources of sharing y variation in the ethical reasoning of managers. Journal of Business Ethics, 42 (2), $101-125$.

Güler A., Asli A. y Ozlem K. (2010). Managing corporate performance: Investigating the relationship between corporate social responsibility and financial performance in emerging markets. International Journal of Productivity and Performance Management, 59 (3), $229-254$

Halila, F. (2007). Networks as a means of supporting the adoption of organizational innovations in SMEs: the case of environmental management systems (EMSS) based on ISO 14001. Corporate Social Responsibility and Environmental Management, 14, 167-181.

Hambrick, D.C. y Mason, P.A. (1984). Upper Echelons: The Organization as a Reflection of Its Top Managers. Academy of Management Review, 9 (2), 193206.

Hammann, E.M., Habisch, A. y Pechlaner, H. (2009). Values that create value: Socially responsible business practices in SMEs - Empirical evidence from German companies. Business Ethics: A European Review, 18 (1), 37-51.

Hazlina, N. y Seet, P. S. (2010). Gender variations in ethical and socially responsible considerations among SME entrepreneurs in Malaysia. International Journal of Business and Society, 11 (1), 77-88.

Herrera Madueño, J., Larrán Jorge, M. y Sánchez Gardey, G. (2011). Effects of family ownership on SME performance. Revista de Empresa Familiar, 1 (2), 5-16.

Herrera, J., Larrán, M. y Sánchez, G. (2012). Una propuesta metodológica para el análisis de la eficiencia de las pequeñas y medianas empresas familiares. Revista Española de Financiación y Contabilidad. 41 (153), 291307.

Herrera, J., Larrán, M. y Martínez-Martínez, D. (2013). Relación entre responsabilidad social y performance en las pequeñas y medianas empresas: Revisión bibliográfica. Cuadernos de Gestión, 13 (2), 3965 .

Husillos, J. y Álvarez-Gil, M. J. (2008). A stakeholder-theory approach to environmental disclosures by small and medium enterprises (SMES). Revista de Contabilidad, 11 (1), 125-156.

Jamali, D., Zanhour, M. y Keshishian, T. (2009). Peculiar Strengths and Relational Attributes of SMEs in the Context of CSR. Journal of Business Ethics, 87 (3), 355-377.

Jenkins, H. (2004). A critique of conventional CSR theory: An SME perspective. Journal of General Management, 29 (4), 37-57.

Jenkins, H. (2006). Small business champions for corporate social responsibility. Journal of Business Ethics, 67, 241-256.

Jenkins, H. (2009). A 'business oportunity' model of corporate social responsibility for small- and mediumsized enterprises. Business Ethics: A European Review, 18 (1), 21-36.

Krauss, A., Rūtelionè, A. y Piligrimienè, Ž. (2010). SME in search for feasible concepts for systematic social responsibility in times of crisis. Economics and Management, 15, 635-641.

Kusyk, S.M. y Lozano, J.M. (2007). Corporate responsibility in small and medium-sized enterprises SME social performance: a four-cell typology of key drivers and barriers on social issues and their implications for stakeholder theory. Corporate Governance, 7 (4), 502515 .

Lahdesmaki, M. (2005). When Ethics Matters Interpreting the ethical discourse of small nature-based entrepreneurs. Journal of Business Ethics, 61, 55-68.

Lepoutre, J. y Heene, A. (2006). Investigating the Impact of Firm Size on Small Business Social Responsibility: A Critical Review. Journal of Business Ethics, 67, 257-273.

Longo, M., Mura, M. y Bonoli, A. (2005). Corporate social responsibility and corporate performance: the case of Italian SMEs. Corporate Governance, 5 (4), 28-42.

Long, R.G. y Mathews, K.M. (2011). Ethics in the Family Firm: Cohesion Through Reciprocity and Exchange. Business Ethics Quarterly, 21(2), 287-308.

Longenecker, J. G., Mckinney, J. A. y Moore, C. W. (1989). Ethics in small business. Journal of Small Business Management, January, 27-31.

López-Gamero, M. D., Molina-Azorín, J. F. y Claver- 
Cortés, E. (2009). The whole relationship between environmental variables and firm performance: Competitive advantage and firm resources as mediator variables. Journal of Environmental Management, 90 (10), 3110-3121.

Luken, R. y Stares, R. (2005). Small Business Responsibility in Developing Countries: A Threat or an Oortunity?. Business Strategy and the Environment, 14 (1), 38-53.

Maxhaam Iii, J. G. y Netemeyer, R. G. (2003): "Firms reap what they sow: the effects of shared values and perceived organizational justice on customer's evaluations of complaint handling", Journal of Marketing, 67, 46-56.

Miller, D. y Le Breton-Miller, I. (2003). Challenge versus advantage in family business. Strategic Organization, 1, 127-134.

Moneva, J. M. y Hernández, J. C. (2009), Responsabilidad social corporativa e información de sostenibilidad en la Pyme. Revista Internacional de la Pequeña y Mediana Empresa, 1 (2), 23-41.

Moore, G. y Spence, L. (2006). Editorial: Responsibility and small business. Journal of Business Ethics, 67 (3), 219-226.

Muñoz, P. y Cabaleiro, R. (2009). Adopción del "balance scorecard" para la gestión de la responsabilidad social y medioambiental de la empresa. Revista de Responsabilidad Social de la Empresa, 2, 77-106.

Muñoz, M.J., Fernández, M.A., Nieto, L., Rivera, J.M. y Escrig, E. (2009). SMEs and corporate social responsibility. The perspective from Spanish companies. International Journal of Sustainable Economy, 1 (3), 270288 .

Murillo, D. y Lozano, J.M. (2006). SMEs and CSR: An approach to CSR in their own Words. Journal of Business Ethics, 67 (3), 227-240.

Bajo, N. y Duran, J.J. (2009). Responsabilidad social y variables estratégicas en las grandes empresas españolas. Boletín Revista de Responsabilidad Social de la Empresa, 2, 227-240.

Nawrocka, D. (2008). Environmental supply chain management, ISO 14001 and RoHS. How are small companies in the electronics sector managing?. Corporate Social Responsibility and Environmental Management, $15,349-360$

Nielsen, A.E. y Thomsen, CH. (2009). Investigation CSR communication in SMEs: a case study among Danish middle managers. Business Ethics: A European Review, 18, (1), 83-93.

Observatorio Europeo De Pymes (2002). European SMEs and Social and Environmental Responsibility (Office for Offical Publications of the European Communities, Luxembourg).

Pearson, K. (1911). On the probability that two independent distributions of frequency are really samples from the same population. Biometrika, 8, 250-254.

Pedersen, E.R. (2009). The many and the few: rounding up the SMEs that manage CSR in the supply chain. An Internacional Journal, 14 (2), 109-116.

Perrini, F., Pogutz, S. y Tencati, A. (2006). Corporate social responsibility in Italy: state of the art. Journal of Business Strategies, 23 (1), 65-91.

Perrini, F., Russo, A. y Tencati, A. (2007). CSR strategies of SMEs and large firms. Evidence from Italy. Journal of Business Ethics, 74 (3), 285-300.

Perrini, F. y Minoja, M. (2008). Strategizing corporate social responsibility: evidence from an Italian mediumsized, family-owned company. Business Ethics: A European Review, 17 (1), 47-63.

Preuss, L. y Perschke, J. (2010). Slipstreaming the Larger Boats: Social Responsibility in Medium-Sized Businesses. Journal of Business Ethics, 92 (4), 531-551.

Quinn, J. (1997). Personal Ethics and Business Ethics: The Ethical Attitudes of Owner/ Managers of Small Business. Journal of Business Ethics, 16, 119-127.

Reyes, L. y Sacristán, M. (2003). Análisis de las diferencias de comportamiento de las empresas familiares cotizadas. Revista Europea de Dirección y Economía de la Empresa, 12 (2), 51-70.

Roberts, S., Lawson, R. y Nicholls, J. (2006). Generating Regional Scale Improvements in SME Corporate Responsibility Performance: Lessons from Responsibility Northwest. Journal of Business Ethics, 67, 275-286.

Rodgers, C. (2010). Sustainable entrepreneurship in SMEs: a case study analysis. Corporate Social Responsibility and Environmental Management, 17, 125132.

Rosener, J.B. (1995) America's Competitive Secret: Utilizing Women as a Management Strate, Oxford University Press, New York.

Rosener, J.B. (1990). Way women lead. Harvard Business Review, 68, 119-125.

Roy, M. J. y Thérin, F. (2008). Knowledge acquisition and environmental commitment in SMEs. Corporate Social Responsibility and Environmental Management, 15, 249-259.

Russo, A. y Tencati, A. (2009). Formal vs. informal CSR strategies: Evidence from Italian micro, small, medium-sized, and large firms. Journal of Business Ethics, 85, 339-353.

Salvato, C. (2002). Towards a stewardship theory of the family firm. Research Forum Proceedings of the family business network, 13th Annual Congress. Helsinky, Finland.

Schulze, W S, Lubatkin, M. H, Dino, R. N y

Herrera, J., Larrán M., Lechuga, M.P. y Martínez, D. (2014). Motivaciones hacia la Responsabilidad Social en las PYMEs familiares. Revista de Empresa Familiar, 4(1), 21-44. 
Buchholtz, A.K. (2001). Agency relationships in family firms: Theory and evidence. Organization Science, 12 (2), 99-116.

Simga-Mugan, C., Daly, B.A., Onkal, D. y Kavut, L. (2005). The influence of nationality and gender on ethical sensitivity: An application of the issue-contingent model. Journal of Business Ethics, 57 (2), 139-159.

Sirmon, D. G. y M. A. Hitt (2003). Managing Resources: Linking Unique Resources, Management, and Wealth Creation in Family Firms. Entrepreneurship: Theory and Practice 27, 339-358.

Spence, L. J. y Rutherfoord, R. (2001). Social responsibility, profit maximisation and the small firm owner-manger. Journal of Small Business and Enterprise Development, 8 (2), 126-139.

Spence, L. y Lozano, F. (2000). Communicating about Ethics with Small Firms: Experiences from the UK and Spain. Journal of Business Ethics. 27 (1), 43-53.

Spence, L.J. y Schmidpeter, R., (2003). SMEs, Social Capital and the Common Good, Journal of Business Ethics. 45 (1-2), 93-108.

Spence, L.J., Jeurissen, R. y Rutherfoord, R. (2000). Small Business and the environment in the UK and the Netherlands: Toward stakeholder cooperation. Business Ethics Quarterly, 10 (4), 945-965.

Spence, L.J., Schmidpeter, R. y Habisch, A. (2003). Assessing social capital: small and medium sized enterprises in Germany and the U.K. Journal of Business Ethics, 47 (1), 17- 29.

Spence, L.J. y Perrini, F. (2009). Practice and politics: Ethics and social responsibility in SMEs in the European Union. African Journal of Business Ethics, 4 (2), 20-31.

Stavrou E., Kassinis G. y Filotheou, A. (2007), Downsizing and Stakeholder Orientation Among the Fortune 500: Does Family Ownership Matter? Journal of Business Ethics, 72 (2), 149-162.

Strautmanis, J. (2008). Employees' values orientation in the context of corporate social responsibility. Baltic Journal of Management, 3 (3), 346-358.

Sweeney, L. (2007). Corporate Social Responsibility in Ireland: barriers and oPortunities experienced by SMEs when undertaking CSR. Dublin Institute of Technology, Dublin, Ireland. Corporate Governance - BradfordEsmerald Group Publishing Limited, 7 (4), 516-523.

Tencati, A., Perrini, F. y Pogutz, S. (2004). New tools to foster socially responsible behavior. Journal of Business Ethics, 53, 173.

Tilley, F (2000). Small Firm Environmental Ethics: How Deep Do They Go?. Business Ethics: A European Review, 9 (1), 31-41.

Udayasankar, K. (2008). Corporate social responsibility and firm size. Journal of Business Ethics, 83 (2), 167-175.
Uhlaner, L. M, Van Goor-Balk, H. J. M. y Masurel, E. (2004). Family business and corporate social responsibility in a sample of Dutch firms. Journal of Small Business and Enterprise Development, 11 (2), 186194.

Van Buren, H. J. III (2005). An employee-centered model of corporate social performance. Business Ethics Quarterly, 15, 687-709.

Villalonga, B. y Amit, R. (2004). How do family ownership, control and management affect firm value? Journal of Financial Economics, 80 (2), 385-417.

Vinnicombe, S. (1995). Essence of Women in Management. Pearson TPR.

Vitell, S.J., Dickerson, E.B. y Festervand, T.A. (2000). Ethical problems, conflicts and beliefs of small business professionals. Journal of Business Ethics, 28 (1), 15-24.

Vives, A., Corral, A., e Isasi, I. (2005). Responsabilidad Social de la Empresa en las Pymes de Latinoamérica (Interamerican Development Bank, Washington, DC).

Vives, A (2006). Social and environmental responsibility in small and medium enterprises in Latin America. The Journal of Corporate Citizenship, 21, 3950 .

Von Weltzien Hoivik, H. y Mele, D. (2009). Can an SME become a global corporate citizen? Evidence from a case study. Journal of Business Ethics, 88, 551-563.

Walls, J. L., Phan, P. y Berrone, P. (2007). A longitudinal study of the link between corporate governance and environmental strategy. Paper presented at the Sixty-Sixth Annual Meeting of the Academy of Management, Philadelphia.

Wagner, M. (2010). Corporate social performance and innovation with high social benefits: A quantitative analysis. Journal of Business Ethics, 94 (4) 581-594.

Ward, J. L. (2004). Perpetuating the family business. 50 lessons learned from long lasting, successful families in business. New York: Palgrave Macmillan.

Webb, E. (2004). An examination of Socially Responsible Firms' Board Structure. Journal of Management and Governance, 8 (3), 255-277.

Williamson, D., Lynch-Wood, G. y Ramsay, J. (2006). Drivers of environmental behaviour in manufacturing SMEs and the implications for CSR. Journal of Business Ethics, 67, 317-330.

Worthington, I., Ram, M. y Jones, T. (2006). Exploring corporate social responsibility in the UK Asian small business community. Journal of Business Ethics, $67,201-217$.

Zellweger, T y Astrachan, J. (2008). On the emotional value of owning a firm. Family Business Review, 21 (4), 347-363. 
Zellweger, T. M. y Nason, R. S. (2008). A stakeholder perspective on family firm performance. Family Business Review, 21, 203-216.

Zhu, Q., Sarkis, J., Lai, K. y Geng, Y. (2008). The role of organizational size in the adoption of green suply chain management practices in China. Corporate Social Responsibility and Environmental Management, 15, 322337.

Zobel, T. (2007). The 'pros' and 'cons' of joint EMS and group certification: a Swedish case study. Corporate Social Responsibility and Environmental Management, 14, 152-166. 


\section{Anexo 1}

Barreras para la implantación y desarrollo de la RSE en las Pymes.

Barreras

Escasez de Recursos. Hace referencia a que las Pymes presentan dos grandes problemas en cuanto a la insuficiencia de recursos financieros y humanos para llevar a cabo una gestión global de la RSE)

Escasez de tiempo disponible para llevar a cabo actividades de RSE

Falta de formación/capacidades, ya que los propietarios/gerentes perciben que no tienen las aptitudes y actitudes necesarias para llevar a cabo este tipo de actividades

Percepción de Costes, los gerentes de las empresas perciben las actividades de RSE como una fuente generadora de costes

No generan valor, la incertidumbre sobre el efecto que tiene estas políticas o prácticas sobre la rentabilidad, ello concuerda con la situación de que la falta de comunicación sea una de las barreras también consideradas.

Falta de presiones, concretamente hace referencia a presiones de los stakeholders tanto internos como externos.

Falta de incentivos o escasez de apoyo por parte de las instituciones, públicas y privadas, para la implantación de la gestión de RSE.

\section{Autor/es}

Vives, 2006; Worthington et al., 2006; Jenkins, 2006; Kusyk y Lozano, 2007; Sweeny, 2007; Zhu et al., 2008; Roy y Thérin, 2008; Bradford y Fraser, 2008; Ciliberti et al., 2009; Gadenne et al., 2009; Preuss, y Perschke, 2010; BosBrouwers, 2010

Jenkins, 2006; Roberts, et al., 2006; Perrini et al., 2006; Worthington et al., 2006; Kusyk y Lozano, 2007; Bradford y Fraser, 2008; Gadenne et al., 2009; Preuss, y Perschke, 2010

Spence y Lozano, 2000; Tilley, 2000; Longo et al., 2005; Jenkins, 2006; Roberts, Lawson y Nicholls, 2006; Perrini et al., 2006; Murillo y Lozano, 2006; Bradford y Fraser, 2008; Zhu et al., 2008; Jamali et al., 2009; Muñoz et al., 2009; Borga et al., 2009; Gadenne et al., 2009; Preuss, y Perschke, 2010; Rodgers, 2010

Jenkins, 2004; Longo et al., 2005; Cabrera et al., 2005; Perrini et al., 2006; Halila, 2007; Bradford y Fraser, 2008; Nawrocka, 2008; Battaglia et al., 2010

Jenkins, 2006; Halila, 2007; Bradford y Fraser, 2008; Zhu et al., 2008; Fassin, 2008; Spence y Perrini, 2009; Muñoz et al., 2009; Nielsen y Thomsen, 2009; Preuss y Perschke, 2010;

Vitell et al., 2000; Lahdesmaki, 2005; Zhu et al., 2008; Ciliberti et al., 2008; Nawrocka, 2008; Spence y Perrini, 2009; Gadenne et al., 2009

Tilley, 2000; Vitell, et al., 2000; Roberts et al., 2006; Halila, 2007; Spence y Perrini, 2009

Friedman et al., 2000; Jenkins, 2006; Ciliberti et al., 2008

Longo et al., 2005; Vives, 2006; Zhu et al., 2008; Sweeney, 2007; Spence y Perrini, 2009; Borga et al., 2009;

Worthington et al., 2006; Roberts et al., 2006; Spence y Perrini, 2009

Lahdesmaki, 2005; Spence y Perrini, 2009

Spence y Lozano, 2000; Vives, 2006; Fassin, 2008; Battaglia et al., 2010

Perrini et al., 2007; Preuss, y Perschke, 2010 
Anexo 2

44 Aceleradores para la impiantación y desarmolio de la RSE en las Pymes 4, no. 1, Mayo 2014

Aceleradores

(a)

Creencias, ética y valores de los propietarios/gerentes

Presiones que pudieran ejercer los stakeholders tanto internos como externos

Percepción de que estas actividades contribuyen a una mejora de la imagen/reputación

Impacto sobre el resultado económico de la empresa ya sea mediante una reducción de costes, sobre un incremento de los ingresos o ambas simultaneamente

Mejora o creación de fuertes vínculos con redes para fomentar la RSE, esto es, capital social

Cumplimiento con la normativa vigente

Nivel de formación que posean $y$ reciban los empleados y los miembros de la alta dirección de la organización

Tamaño de la empresa en cuanto a la flexibilidad que tienen las Pymes para daptarse a los requerimientos del mercado

Otros aceleradores como, por ejemplo, el sector de pertenencia, la motivación de los empleados, la influencia de los miembros de la familia
Autor/es

Spence y Lozano, 2000; Vitell et al., 2000; Spence y Rutherfoord, 2001; Longo et al., 2005; Vives, 2006; Lahdesmaki, 2005; Perrini et al., 2006; Fuller y Tian, 2006; Murillo y Lozano, 2006; Worthington et al., 2006; Kusyk y Lozano, 2007; Perrini y Minoja, 2008; Jamali et al., 2009; et al., 2009; Jenkins, 2009; Gadenne et al., 2009; Nielsen y Thomsen, 2009; Hammann et al., 2009; Von Weltzien Hoivik y Mele, 2009; Baden et al., 2010; Rodgers, 2010

Spence y Lozano, 2000; Jenkins, 2004; Uhlaner et al., 2004; Vives, 2006; Luken y Stares, 2005; Williamson et al., 2006; Perrini et al., 2006; Jenkins, 2006; Sweeney, 2007; Bradford y Fraser, 2008; Roy y Thérin, 2008; Ciliberti et al., 2008; Zhu et al., 2008; Nawrocka, 2008; Husillos y Álvarez-Gil, 2008; Jamali et al., 2009; Ciliberti et al., 2009; Muñoz et al., 2009; Jenkins, 2009; Gadenne et al., 2009; Preuss, y Perschke, 2010; Baden et al., 2010

Jenkins, 2004; Longo et al., 2005; Cabrera et al., 2005; Perrini et al., 2006; Jenkins, 2006; Fuller y Tian, 2006; Kusyk y Lozano, 2007; Husillos y Álvarez-Gil, 2008; Gadenne et al., 2009; Borga et al., 2009; Nielsen y Thomsen, 2009

Spence et al., 2000; Cabrera et al., 2005; Williamson et al., 2006; Jenkins, 2006; Roberts et al., 2006; Halila, 2007; Borga et al., 2009; Von Weltzien Hoivik y Mele, 2009; Nielsen y Thomsen, 2009; Gadenne et al., 2009; Preuss, y Perschke, 2010

Spence et al., 2000; Spence y Lozano, 2000; Spence y Schmidpeter, 2003; Spence et al., 2003; Graafland et al., 2003; Longo et al., 2005; Roberts et al., 2006; Worthington et al., 2006; Perrini et al., 2006; Fuller y Tian, 2006; Halila, 2007; Kusyk y Lozano, 2007; Zobel, 2007; Sweeney, 2007; Roy y Thérin, 2008; Borga et al., 2009; Jenkins, 2009; Nielsen y Thomsen, 2009; Murillo y Lozano, 2009; Von Weltzien Hoivik y Mele, 2009; Fisher et al., 2009; Bos-Brouwers, 2010; Davies y Crane, 2010

Spence y Lozano, 2000; Tilley, 2000; Friedman et al., 2000; Vitell et al., 2000; Vives, 2006; Williamson et al., 2006; Murillo y Lozano, 2006; Fassin, 2008; Nawrocka, 2008; Zhu et al., 2008; Bradford y Fraser, 2008; Hammann et al., 2009; Von Weltzien Hoivik y Mele, 2009; Baden et al., 2010; Krauss et al., 2010

Spence y Lozano, 2000; Zobel, 2007; Bradford y Fraser, 2008; Zhu et al., 2008; Jenkins, 2009; Preuss y Perschke, 2010

Spence y Lozano, 2000; Lahdesmaki, 2005; Bradford y Fraser, 2008; Pedersen, 2009; Russo y Tencati, 2009

Serwinek, 1992; Quinn, 1997; Spence y Lozano, 2000; Uhlaner et al., 004; Vives, 2006; Murillo y Lozano, 2006; Jenkins, 2006; Worthington et al., 2006; Bradford y Fraser, 2008; Jamali et al., 2009; Nielsen y Thomsen, 2009 